\title{
Dissociating conscious and unconscious influences on visual detection effects
}

\author{
Timo Stein ${ }^{1 \star}$ and Marius V. Peelen ${ }^{2}$ \\ ${ }^{1}$ Brain and Cognition, Department of Psychology, University of Amsterdam, , The Netherlands \\ ${ }^{2}$ Donders Institute for Brain, Cognition and Behaviour, Radboud University, The Netherlands
}

*Corresponding author: Timo Stein (timo@timostein.de) 


\begin{abstract}
The scope of unconscious processing is highly debated, with recent studies showing that even highlevel functions such as perceptual integration and category-based attention occur unconsciously. For example, upright faces that are suppressed from awareness through interocular suppression break into awareness more quickly than inverted faces. Similarly, verbal object cues boost otherwise invisible objects into awareness. Here, we replicate these findings, but find that they reflect a general difference in detectability not specific to interocular suppression. To dissociate conscious and unconscious influences on visual detection effects, we use an additional discrimination task to rule out conscious processes as a cause for these differences. Results from this detection-discrimination dissociation paradigm reveal that while face orientation is processed unconsciously, category-based attention requires awareness. These findings provide insights into the function of conscious perception and offer an experimental approach for mapping out the scope and limits of unconscious processing.
\end{abstract}

\title{
Main
}

A thriving field of research in psychology and neuroscience has made progress in revealing the putative functions of consciousness ${ }^{1-4}$. Influential theories of consciousness converge on the notion that while much mental activity is unconscious, consciousness is required for more complex and flexible operations, such as integration over space and time, object recognition, control of voluntary attention, and intentional action ${ }^{3,5,6}$. Recently, this view has been challenged by studies showing that many highlevel operations ${ }^{7}$, including perceptual integration ${ }^{8}$, attention ${ }^{9}$, working memory ${ }^{10}$, and executive control $^{11}$, can happen unconsciously. Such findings beg the question why consciousness emerged at all in the course of evolution. However, such conclusions may be premature, as much of the evidence for high-level unconscious processing is highly debated, with controversies revolving around how to manipulate and measure unconscious processing ${ }^{12-15}$. Here, we first show that one of the most widely adopted behavioural approaches, the "breaking continuous flash suppression" (b-CFS) paradigm, cannot provide evidence for unconscious processing. We then present an alternative approach and demonstrate how it can distinguish between functions that do and do not require consciousness.

Many studies claiming high-level unconscious processing have investigated differences in the speed of stimulus detection, with the assumption that differences in detection speed reflect differential unconscious processing preceding detection ${ }^{16-18}$. It is widely recognized, however, that a detection difference can arise at any stage between stimulus (retina) and response: Detection responses could be influenced by later conscious processes (e.g. related to stimulus recognition and identification) or by 
non-perceptual factors (e.g. decisional processes and response biases) $)^{19,20}$. In other words, detection differences could reflect either unconscious or conscious processing. As explained in more detail below, the $b$-CFS paradigm ${ }^{16,18,20,21}$ appeared to have overcome this problem by comparing detection differences during $\mathrm{CFS}^{22}$, a strong interocular suppression technique, to detection differences in control paradigms not involving CFS, thereby dissociating conscious and unconscious contributions to detection effects. To date, findings from more than $120 \mathrm{~b}$-CFS studies have changed our view of which functions can occur unconsciously $^{21}$. Examples include face perception ${ }^{23,24}$ and person evaluation ${ }^{25}$, threat detection ${ }^{26,27}$ and emotion recognition ${ }^{28}$, perception of visual illusions ${ }^{29}$ and causality ${ }^{30}$, perceptual and multimodal integration $^{31-35}$, control of attention ${ }^{36-38}$, working memory ${ }^{39,40}$, comprehension of syntax ${ }^{41}$, meaning ${ }^{42,43}$, language $e^{44,45}$, and reading ${ }^{46}$.

Here, focusing on unconscious processing of face orientation ${ }^{18,47,48}$, we first show that the b-CFS approach cannot isolate unconscious processing, reporting similarly strong face inversion detection effects in b-CFS and multiple carefully designed control paradigms. This leaves open the question whether these effects are conscious or unconscious. As a solution, we use an additional discrimination task to rule out conscious processes as a cause for differences in stimulus detection. Applied to face detection, we report a face inversion effect even when observers were unable to tell whether the stimuli were upright or inverted faces, providing unequivocal evidence for an unconscious origin of the effect. We then investigated whether the effect of attention at the level of object category modulates stimulus processing unconsciously. Similar to face inversion, such effects have previously been reported with b$\mathrm{CFS}^{37,38,44,45}$, suggesting an unconscious origin. In contrast to face inversion, however, we found that the effect of attention required awareness, such that attention modulated stimulus detection only when participants were aware of the validity of the attention cue. Together, these findings demonstrate that seemingly similar effects on detection can reflect distinct conscious or unconscious processes. More generally, we show how conscious and unconscious contributions to such detection effects can be dissociated, equipping scientists with a simple experimental approach to study the scope and limits of unconscious processing.

To determine whether detection effects obtained with b-CFS reflect unconscious processing during interocular suppression, we first tested whether similar effects can be obtained with other detection paradigms not involving interocular suppression. In CFS, dynamic masks flashed into one eye suppress visibility of a stimulus presented to the other eye for up to several seconds ${ }^{22}$. Studies that tested unconscious processing of stimuli rendered fully invisible through CFS, for example by measuring priming effects, brain or psychophysiological responses, have yielded conflicting results ${ }^{17,49}$, sparking heated debates about the existence of high-level unconscious processing ${ }^{7,12,50-52}$. For example, while two 
studies found that invisible face stimuli evoked face-specific electrophysiological responses ${ }^{53,54}$, a more recent study with greater statistical power failed to detect any signal to suppressed faces ${ }^{55}$. Similarly, faces rendered invisible through CFS typically fail to induce adaptation aftereffects ${ }^{56-58}$. However, with the development of CFS, another, fundamentally different approach, b-CFS, has now consistently produced evidence for high-level unconscious processing. In the b-CFS paradigm, localization or detection tasks are used to measure the time it takes a stimulus to "break" CFS and become visible. Differences between conditions in breaking suppression time are thought to reflect differences in unconscious processing the stimuli received while still being suppressed ${ }^{16,18,21}$. This reasoning assumes an "all-or-none" transition between unconscious and conscious processing, and a precise measure of the transition point. Neither of these assumptions have been validated in the b-CFS paradigm, and both are unlikely to be correct ${ }^{21}$. For example, there is reason to believe that visual awareness is a gradual rather than a binary phenomenon ${ }^{59,60}$. Thus, rather than reflecting enhanced unconscious processing, a detection advantage could reflect enhanced conscious processing, such as stronger pre-activation ${ }^{61}$, faster recognition, identification, or lower response criterion ${ }^{19-21}$.

To rule out conscious processing as a cause of detection differences, b-CFS studies often compared effects with a control paradigm not involving CFS. In this control paradigm stimuli are typically faded in on top of the masks, and masks and stimuli are presented to the same eye, such that no interocular suppression is induced. When a detection difference is obtained with b-CFS but not in the control paradigm, this is taken as evidence that the effect is caused by unconscious processing that occurred while stimuli were suppressed through interocular suppression. For example, in the first b-CFS study ${ }^{18}$, faster detection of upright than inverted faces was found only with b-CFS but not in the control paradigm, indicating that the effect was caused by unconscious processing of face orientation. Thus, the absence of effects in the control paradigm is used to support claims for unconscious processing during CFS. One concern with this logic is that control paradigms may not be optimally sensitive to general differences in conscious stimulus processing. For example, faces faded-in on top of the CFS masks can often be detected based on their contour alone, before inner facial parts become visible (see Supplementary Information). Indeed, in virtually all b-CFS studies, no face inversion effects were obtained in the control paradigm ${ }^{19}$. Furthermore, control paradigms may not be fully comparable to the b-CFS paradigm. For example, response times and presentation durations are usually much longer and more variable in b-CFS than in the control paradigms ${ }^{20}$. 
a

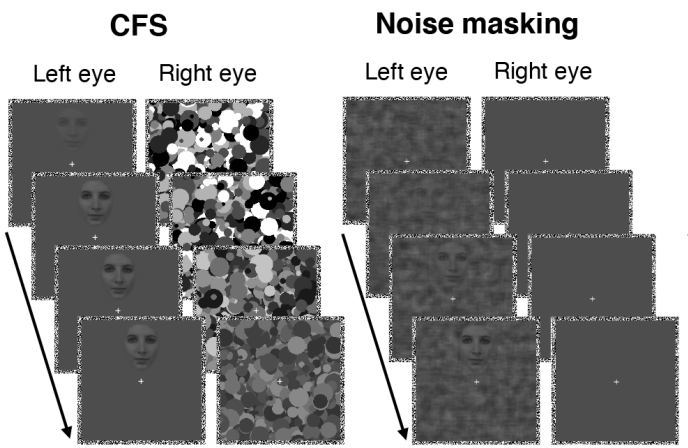

Monoptic masking

Dichoptic masking

Left eye Right eye

Left eye Right eye
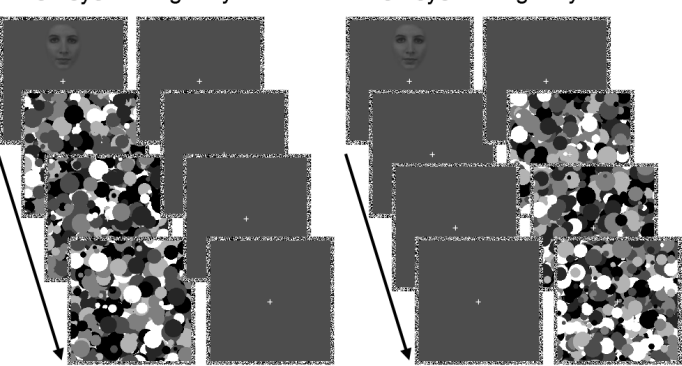

b
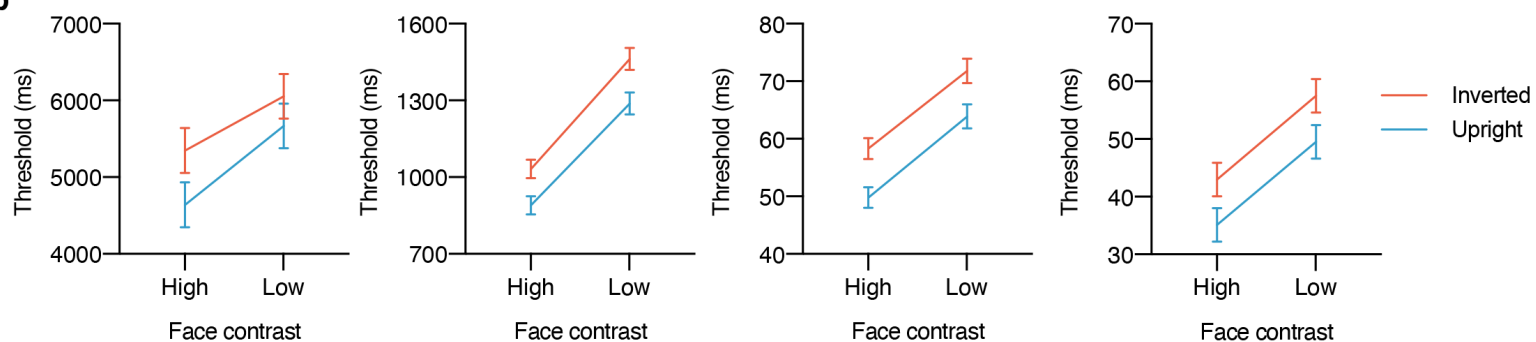

d
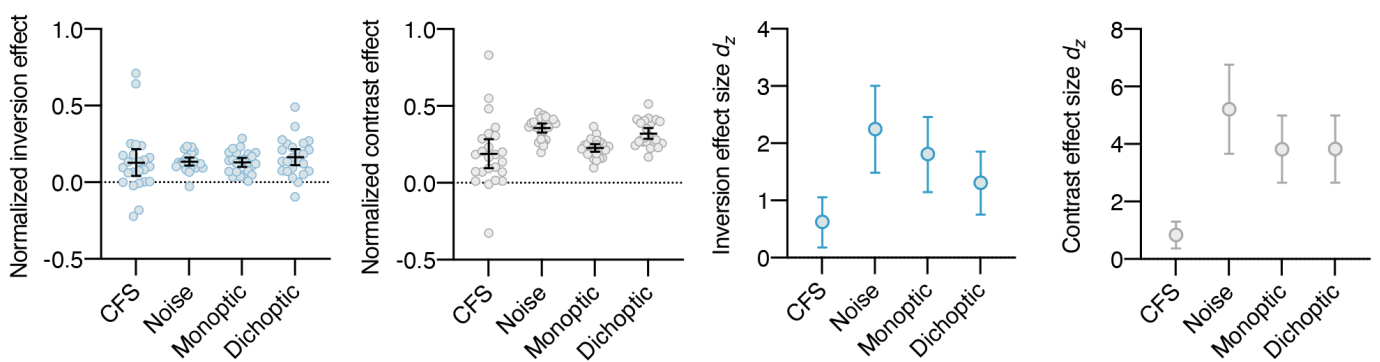

Figure 1. Experiment 1, comparing b-CFS with three other detection paradigms. (a) Schematic trials from the four paradigms. Upright or inverted face stimuli were presented for fixed presentation times, controlled by adaptive staircases. Participants performed non-speeded 4-AFC localization tasks. In CFS, a face was presented to one eye and circular masks, changing every $100 \mathrm{~ms}$, were presented to the other eye. In simultaneous noise masking, a face was presented to the same eye as the phase-scrambled noise masks, which were also changing every $100 \mathrm{~ms}$. In simultaneous noise masking no stimulus was presented to the other eye, thus no interocular suppression was induced. In monoptic backward masking a face was presented briefly to one eye and then masked by three masks presented to the same eye. In dichoptic backward masking, the face stimulus and the masks were presented to different eyes. (b) Mean localization thresholds for low- and highcontrast upright and inverted faces from the four detection paradigms. Note the different scales. Error bars represent $95 \% \mathrm{Cls}$ for the respective comparison between upright and inverted faces. (c) Inversion effects (left panel) and contrast effects (right panel) after latency-normalization. Circles represent individual participants $(N=24)$, horizontal lines the means, and error bars $95 \% \mathrm{Cls}$. (d) Inversion effects (left panel) and contrast effects (right panel) in standardized effect sizes $d_{z}$. Error bars represent $95 \% \mathrm{Cls}$. 


\section{Results}

\section{No advantage of breaking CFS over other detection paradigms}

Our first goal was therefore to test whether b-CFS-like effects can similarly be observed without interocular suppression when using more comparable control paradigms. We took the well-established b-CFS face-inversion effect as a test case, comparing localization of upright and inverted faces. In Experiment 1, we compared b-CFS to three non-CFS paradigms (Figure 1a): 1) Simultaneous noise masking, where the face stimulus was faded-in on top of changing phase-scrambled noise masks (for more details on the rationale see Supplementary Discussion and Supplementary Figure 1);2) backward masking in its monoptic form and 3) backward masking in its dichoptic form, using the same masks as used in CFS.

To achieve optimal sensitivity for detecting differences in all paradigms, we used staircases to adjust presentation durations (1-up, 3-down), separately for each condition and paradigm. This staircase procedure ensures that overall performance is not at floor or ceiling and that it is similar across conditions and paradigms. Accordingly, staircase values (i.e., localization thresholds) were used as a measure of detectability. To rule out decisional influences such as response biases, rather than measuring response times we used the same accuracy-based, non-speeded 4-AFC localization task in all paradigms (see Supplementary Discussion for a discussion of accuracy-based vs. response-time based measures). In addition to manipulating face orientation, we also manipulated luminance contrast, presenting faces with high or low contrast. High-contrast stimuli should have lower localization thresholds than lowcontrast stimuli in all paradigms, reflecting bottom-up stimulus strength. The magnitude of this effect may nonetheless vary across paradigms, reflecting factors of non-interest such as the duration and variability of presentation duration. The contrast manipulation therefore served as a benchmark for the comparison of face inversion effects across paradigms.

For all paradigms we found strong evidence for effects of contrast and face-inversion (all $t(23)>4.35, p$ $<.001, d_{z}>0.88, \mathrm{BF}_{10}>128.13$; Figure 1b; for subject-level display of localization thresholds, refer to Extended Data Figure 1). The very different temporal characteristics of the different paradigms were reflected in localization thresholds and raw effects that differed in orders of magnitude. For example, the overall localization threshold was $5428 \mathrm{~ms}$ in CFS but only $46 \mathrm{~ms}$ in dichoptic backward masking. The effects of contrast and inversion were similarly scaled. For example, in CFS the localization threshold for high-contrast faces was $869 \mathrm{~ms}$ lower than for low-contrast faces, whereas in dichoptic backward masking this difference was just $15 \mathrm{~ms}$. These overall differences reflect the radically different temporal characteristics of the four paradigms. To account for these overall differences, we normalized the contrast and inversion effects by dividing these values by the overall localization threshold for a given 
paradigm, yielding proportional differences in localization thresholds. This analysis revealed that inversion effects were of comparable size in all paradigms $\left(F(3,69)=0.41, p=.75, \eta_{p}{ }^{2}=.02, \mathrm{BF}_{01}=10.86\right)$; contrast effects tended to be larger in simultaneous noise masking than in the other paradigms (Figure 1c). Effects in CFS were more variable than in the other paradigms, such that standardized effect sizes for both contrast and inversion were smaller in CFS than in the other paradigms (Figure 1d).

a

CFS

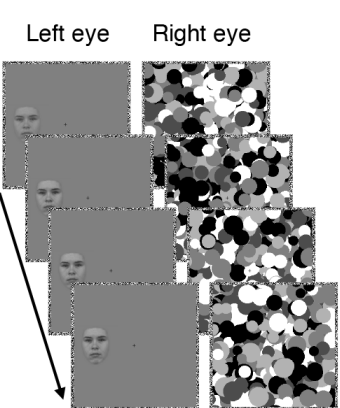

b

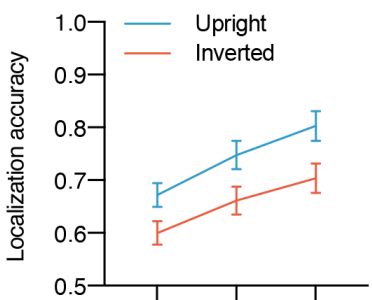

Backward masking

RSVP

Both eyes

Both eyes
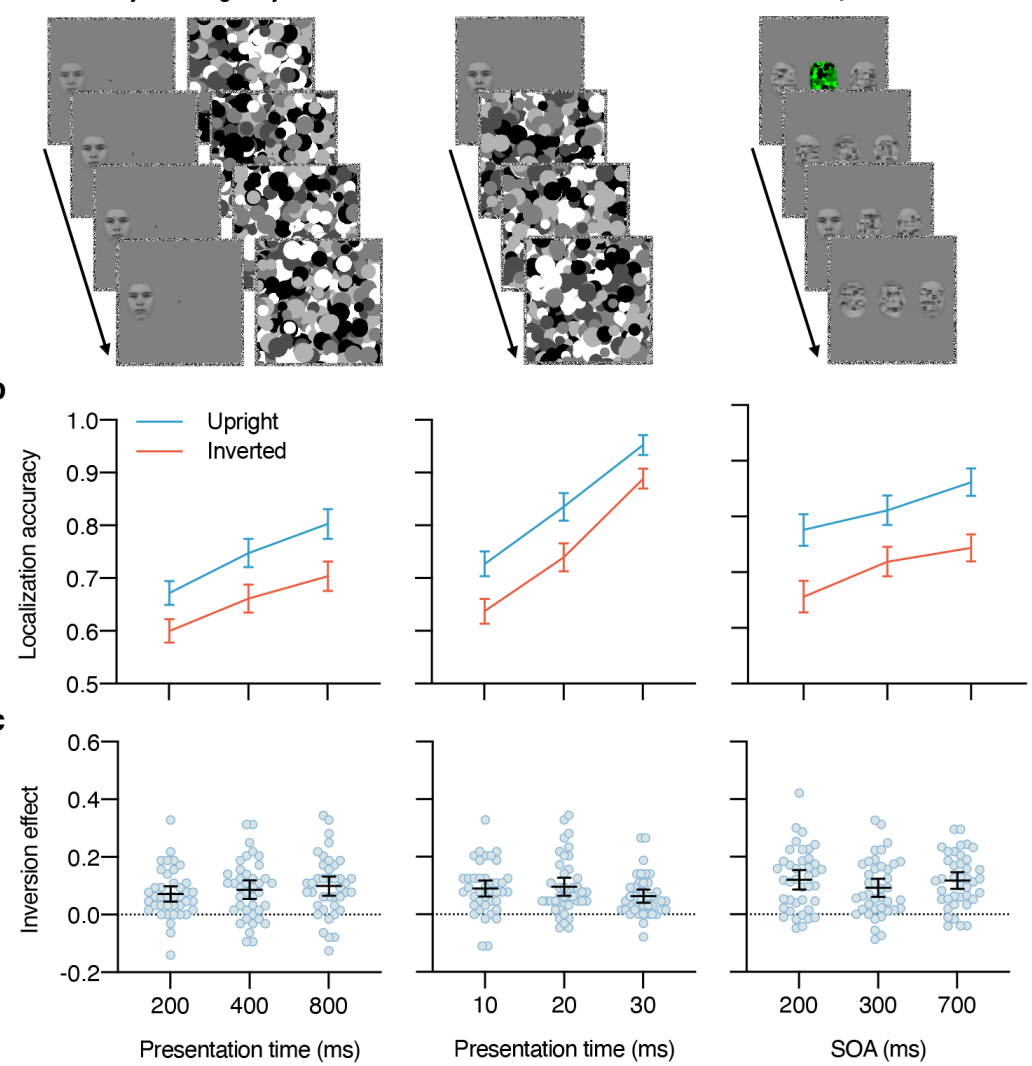

Figure 2. Experiment 2, comparing b-CFS with two other detection paradigms. (a) Schematic trials from the three paradigms. Upright or inverted face stimuli were presented for three different fixed presentation times. Participants performed non-speeded 2-AFC localization tasks. CFS and standard backward masking were similar to Experiment 1. In the rapid serial visual presentation (RSVP) paradigm, stimuli were embedded in three streams of rapidly changing distracters (scrambled faces). Faces were presented in the left or right stream, at different SOAs relative to a first target in the central stream which subjects had to identify (green circles vs. green diamonds). (b) Mean localization accuracy for upright and inverted faces for the three different detection paradigms and presentation times. Error bars represent 95\% Cls for the respective comparison between upright and inverted faces. (c) Inversion effects for the different paradigms and presentation conditions. Circles represent individual participants $(N=40)$, horizontal lines the means, and error bars $95 \%$ Cls. 
To substantiate these findings, in Experiment 2 we compared face inversion effects between CFS, backward masking (with standard binocular viewing), and rapid serial visual presentation (RSVP). Instead of recording staircase thresholds, here we measured detection of stimuli presented for fixed durations using a non-speeded 2-AFC localization task. This was done to generalize the findings and because staircasing could not be implemented in the RSVP paradigm. In CFS, stimuli were presented for 200, 400, or $800 \mathrm{~ms}$; in backward masking for 10,20, or $30 \mathrm{~ms}$, reflecting the paradigms' different temporal characteristics. In the RSVP paradigm, stimuli were embedded in three streams of distracters changing every 100 ms. Faces were presented with three different temporal delays (200, 300, or $700 \mathrm{~ms}$ ) relative to a first target that participants had to identify (Figure 2a). At short temporal delays, face localization is thought to be impaired because attention is still engaged with the first target ${ }^{62}$. Including this attentional blink-like paradigm allowed us to test whether effects would differ between paradigms where detectability is reduced by degrading the input (CFS and masking) and a paradigm where visibility is reduced through inattention (RSVP) ${ }^{63,64}$. However, inversion effects did not differ significantly between paradigms (mixed ANOVA, two-way interaction, $F(2,117)=1.82, p=.17, \eta_{p}^{2}=.03, \mathrm{BF}_{01}=3.25$ ) or presentation conditions (all $F(2,78)<1.75, p>.18, \eta_{p}^{2}<.05, \mathrm{BF}_{01}>4.38$; Figure $2 c$, for subject-level display of localization accuracy, refer to Extended Data Figure 2). For all three paradigms results revealed strong evidence for face-inversion effects (all $t(39)>4.35, p<.001, d_{z}>0.88, \mathrm{BF}_{10}>4.95 \times 10^{6}$; Figure $2 b)$.

Thus, contrary to previous b-CFS studies that typically failed to obtain effects in the control paradigm $^{18,47,48,65}$, we found similar effects in all four non-CFS detection paradigms included in Experiments 1 and 2. This implies that the comparison of b-CFS with control paradigms cannot dissociate conscious and unconscious processing. If a CFS-specific effect is obtained, this may reflect the insensitivity of standard control paradigms to general differences in stimulus detectability rather than CFS-specific unconscious processing.

While our results do not show any CFS-specific effects, this does not necessarily argue against the possibility that effects revealed with visual detection paradigms reflect unconscious processing. In a trivial sense, unconscious processing always precedes stimulus detection (e.g. phototransduction, activity in the optic nerve). However, this does not imply that a difference in stimulus detectability is mediated by differential unconscious processing (Figure 3a). Some differences most likely arise at an early, possibly unconscious stage of visual processing. For example, the effect of stimulus contrast studied in Experiment 1 may involve enhanced firing already at an unconscious, retinal processing stage. For higher-level effects, such as the effect of face inversion, it is unknown at what stage between stimulus 
and response the detection difference arises. In the next section we introduce a paradigm that allows for dissociating conscious and unconscious contributions to visual detection effects.

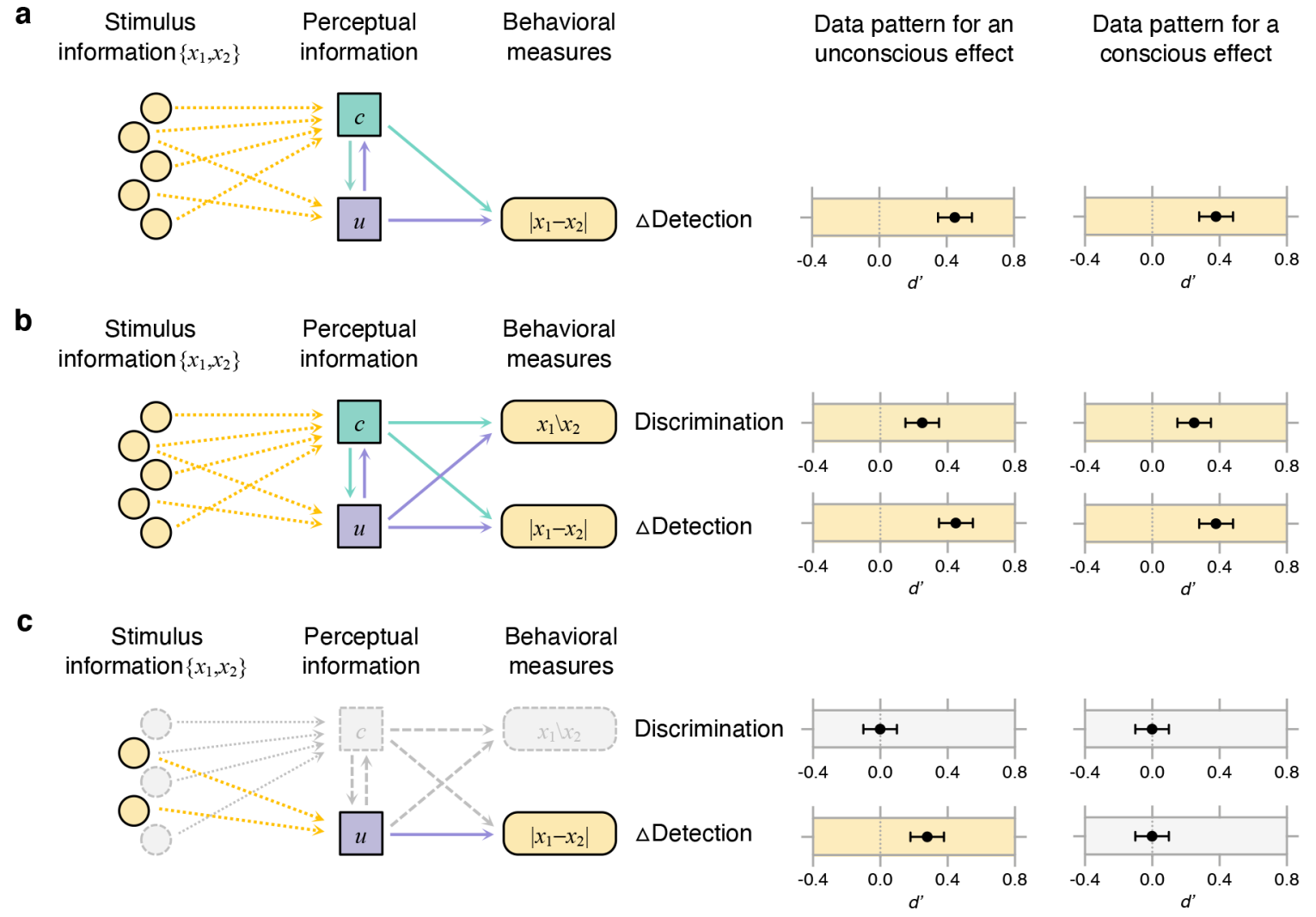

Figure 3. Dissociating conscious and conscious contributions to detection effects. (a) In conventional detection paradigms, a detection difference ( $\Delta$ Detection) between experimental conditions $\left\{x_{1}, x_{2}\right\}$ (e.g. upright vs. inverted faces, validly vs. invalidly cued objects) is often interpreted as reflecting unconscious processing differences $u$. However, $\Delta$ Detection is not an exclusive measure of $u$, but could also reflect differences in conscious processing c. An additional measure is required to rule out conscious processing as a cause for $\Delta$ Detection. As this measure should be exhaustive for a potential conscious processing difference ${ }^{66,67}$, the task needs to measure discriminability of the specific stimulus dimension $\left\{x_{1}, x_{2}\right\}$ that is yielding $\Delta$ Detection (e.g. discrimination of upright vs. inverted faces, discrimination of validly vs. invalidly cued objects). (b) When this discrimination measure yields above-chance performance, conscious processing cannot be ruled out, and $\Delta$ Detection cannot be said to reflect unconscious processing. (c) When the discrimination measure yields chance performance, conscious processing can be ruled out, and $\Delta$ Detection can be interpreted as reflecting unconscious processing differences. These scenarios are illustrated with idealized data patterns providing evidence for an unconscious or a conscious origin of $\Delta$ Detection, respectively. The figure was inspired by Figure 1 in Schmidt and Vorberg $(2006)^{66}$. 


\section{The detection-discrimination dissociation paradigm}

To link a detection difference to differential unconscious processing, an additional measure needs to demonstrate absence of awareness. Without a gold standard for measuring consciousness, the awareness measure should be exhaustive for the critical stimulus dimension underlying the effect ${ }^{66,67}$ (Figure $3 b$ ). The idea is simple: In addition to the detection task, a second task measures awareness of the critical dimension causing the detection difference (e.g. discrimination of upright vs. inverted faces). Absence of awareness is established when participants cannot discriminate this critical dimension. This approach has previously been adopted to demonstrate that a non-discriminable stimulus dimension can cause unconscious priming effects ${ }^{\text {e.g.68,69. }}$. Similarly, any detection difference caused by this dimension (e.g. better detection of upright than inverted faces) must reflect unconscious processing differences (Figure $3 c)$. We adopt this approach to test whether the face inversion effect on stimulus detection, as reported in the previous section, is mediated by conscious or unconscious processes.

Although this "detection-discrimination dissociation" approach can in principle be implemented with any method for presenting visual stimuli (e.g. CFS or backward masking), it requires carefully calibrated presentation parameters that yield chance performance in the discrimination task (to establish absence of awareness) but above-chance performance in the detection task (a prerequisite for measuring differences between conditions). As the strength of CFS varies strongly between and within participants ${ }^{70-73}$, in practice methods other than CFS may be preferable for finding appropriate stimulation parameters. Here, we used (standard) backward masking, where stimulation parameters can be more easily calibrated to present stimuli with maximum bottom-up stimulus strength just below the discrimination threshold. Note that we adopted backward masking rather than CFS only for this technical reason, and we do not wish to imply that our findings are specific to backward masking.

\section{Face orientation can be processed unconsciously}

To test whether detection-discrimination dissociations can provide evidence for unconscious processing of face orientation, in Experiment 3 we measured localization and detection of upright and inverted faces while simultaneously recording participants' ability to distinguish between upright and inverted faces. Face stimuli were presented in two thirds of the trials (one third stimulus-absent trials), for five different presentation times ranging from 8 to $33 \mathrm{~ms}$, and backward masked (Figure 4a). On every trial, participants performed a 2-AFC localization task, reported awareness of the stimulus (on the Perceptual Awareness Scale $[\mathrm{PAS}]^{74}$ ), and indicated face orientation (upright vs. inverted; with the order of the three tasks counterbalanced between participants). To directly compare localization, detection, and discrimination on the same scale, responses from all three tasks were transformed to the signal-detection theory sensitivity index $d$ ' (detection sensitivity was calculated from the "seen" vs. "unseen" responses 
on the PAS in stimulus-present vs. -absent trials, see Methods). Across all presentation times, upright faces were localized and detected better than inverted faces (both $F(1,93)>333.81, p<.001, \eta_{p}{ }^{2}>.78$, $\mathrm{BF}_{10}>8.42 \times 10^{26}$; Figure $4 \mathrm{~b}$, for subject-level display of localization, detection and discrimination sensitivity, refer to Extended Data Figure 3). Inversion effects were larger at intermediate presentation times than at the shortest and longest presentation times (interaction for localization and detection sensitivity, both $F(4,372)>37.78, p<.001, \eta_{p}{ }^{2}>.28, \mathrm{BF}_{10}>9.72 \times 10^{15}$; Figure $\left.4 \mathrm{c}\right)$.

To test for a dissociation between detection and discrimination with maximum bottom-up stimulus strength, we determined the longest presentation time that yielded null sensitivity in the discrimination task but above-chance sensitivity in the localization and detection tasks. At the second shortest presentation time ( $8 \mathrm{~ms}$, followed by a blank of $8 \mathrm{~ms}$ ), orientation discrimination did not differ significantly from chance $\left(t(93)=0.91, p=.18\right.$ (one-tailed), $d_{z}=0.09, \mathrm{BF}_{0+}=3.59$ ), while upright and inverted faces were localized and detected with above-chance performance (all $t(93)>4.63, p<.001, d_{z}$ $>0.47, \mathrm{BF}_{10}>1.40 \times 10^{3}$; Figure $3 \mathrm{~d}$ ). Critically, both localization and detection sensitivity were higher for upright faces than for inverted faces $(M=0.16, S D=0.37$, and $M=0.19, S D=0.40$, respectively; both $t(93)>4.11, p<.001, d_{z}>0.42, \mathrm{BF}_{10}>220.58$; Figure $4 \mathrm{c}$ and $\left.4 \mathrm{~d}\right)$. Thus, face orientation influenced detectability while participants had no conscious access to this dimension, providing evidence for an unconscious origin of the effect. Moreover, the inversion effects in detection and localization also exceeded orientation discrimination when compared directly on the same scale (detection, $t(93)=3.21$, $p=.002, d_{z}=0.33, \mathrm{BF}_{10}=13.16$; localization, $\left.t(93)=2.82, p=.006, d_{z}=0.29, \mathrm{BF}_{10}=4.67\right)$, thereby satisfying an additional criterion for demonstrating unconscious processing ${ }^{66,75}$.

Finally, if the face inversion effect was indeed independent of the ability to discriminate face orientation, it should not differ between trials with correct and incorrect discrimination responses. We tested this in 64 (out of 94) participants who had enough data points with correct and indirect discrimination responses for every condition (excluding the longest presentation time where discrimination performance was at ceiling, see Methods). Supporting our prediction, localization and detection accuracy for upright faces were significantly better than for inverted faces for both trials with correct and trials with incorrect discrimination responses (localization: both $F(1,63)>54.37, p<.001, \eta_{p}^{2}>.46$, $\mathrm{BF}_{10}>1.17 \times 10^{5}$; see Figure 4e; detection: both $\left.F(1,63)>88.82, p<.001, \eta_{p}^{2}>.58, \mathrm{BF}_{10}>1.23 \times 10^{12}\right)$. Inversion effects even tended to be somewhat larger when discrimination was incorrect (interaction, localization: $F(1,63)=4.57, p=.036, \eta_{p}{ }^{2}=.07$, but $\mathrm{BF}_{10}=0.94$; detection: $F(1,63)=3.39, p=.070, \eta_{p}{ }^{2}=$ .05 , but $\mathrm{BF}_{10}=1.65$ ). In other words, the face inversion effect can be dissociated from face orientation discrimination, indicating that the face inversion effect in visual detection is (at least partly) mediated by unconscious processes. 
a

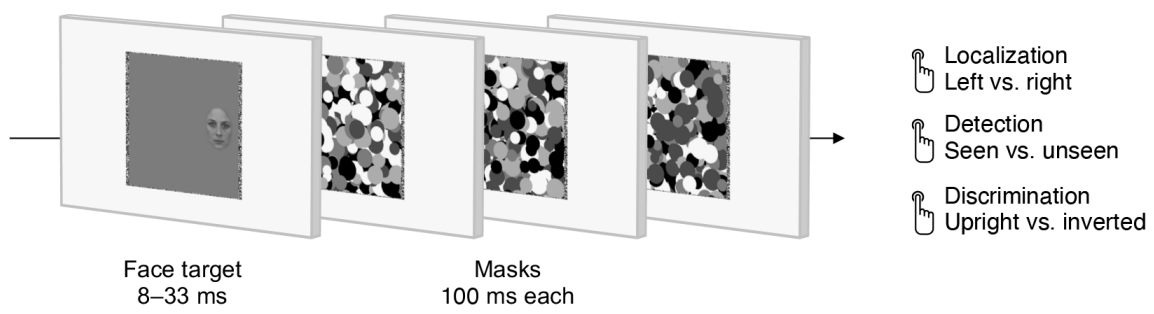

b
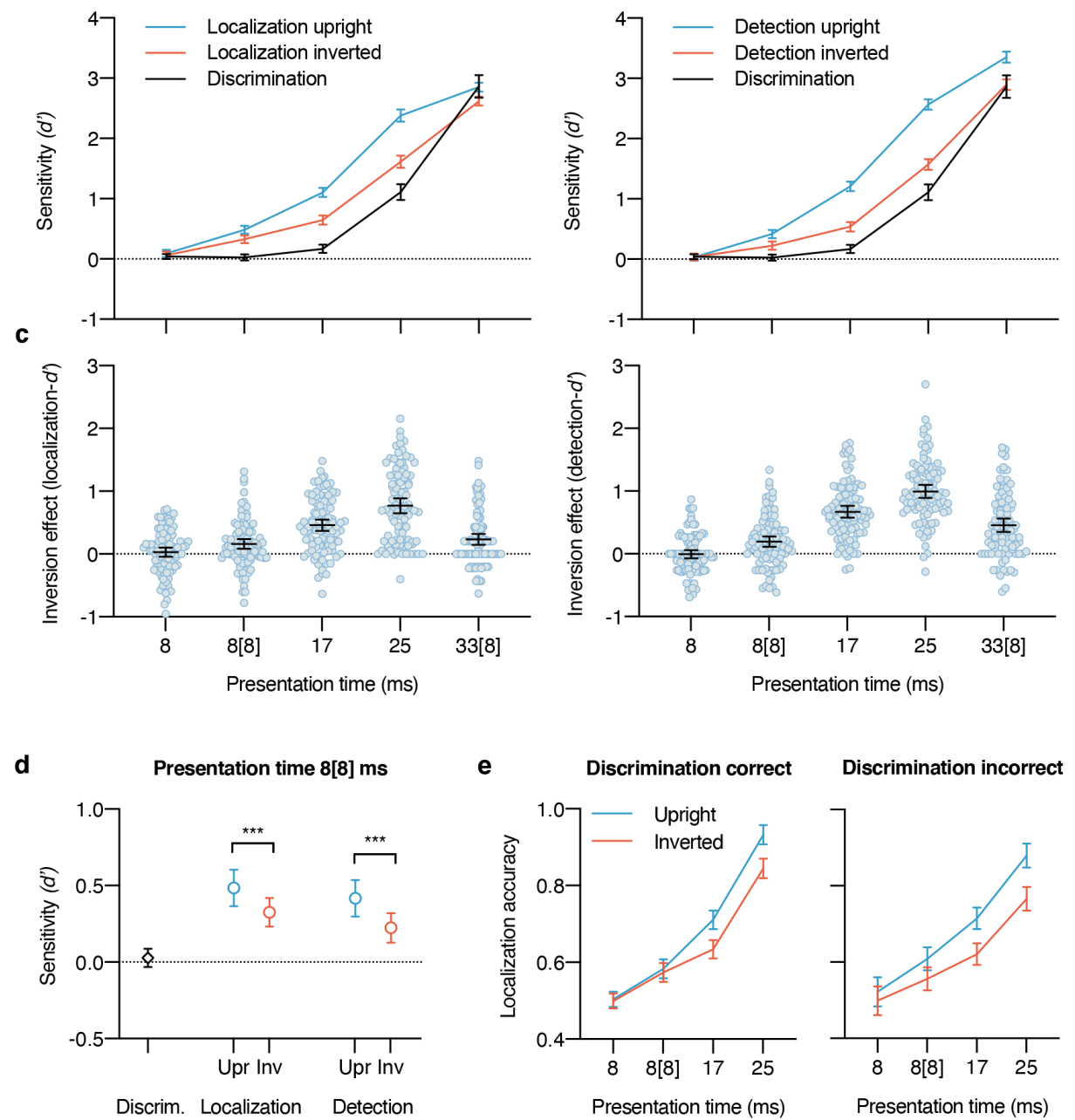

Figure 4. Experiment 3, testing unconscious processing of face orientation with the detectiondiscrimination dissociation approach. (a) Upright or inverted faces were presented for five different fixed presentation times and backward masked (face presentation $8,8[8], 17,25$, or $33[8] \mathrm{ms}$, with the value in square brackets referring to the duration of an additional blank screen between face stimulus and mask). On every trial, participants performed three tasks (order counterbalanced between participants) measuring face localization (2-AFC), face detection (comparing "seen" vs. "unseen" responses on the PAS in face-present vs. absent trials), and discrimination of upright and inverted faces. Responses from all three tasks were transformed to the signal-detection theory sensitivity index $d^{\prime}$. (b) Mean localization sensitivity (left panel) and mean detection sensitivity (right panel) for upright and inverted faces for the five different presentation times (values in square brackets refer to an additional blank screen of $8 \mathrm{~ms}$ between the face stimulus and the mask). Error bars represent $95 \% \mathrm{Cls}$ for the respective comparison between upright and inverted faces. For 
comparison, both panels also show mean sensitivity and 95\% Cls for discriminating between upright and inverted faces for every presentation time. (c) Inversion effects from the localization task (left panel) and from the detection task (right panel), separately for every presentation time. Circles represent individual participants $(N=94)$, horizontal lines the means, and error bars $95 \% \mathrm{Cls}$. (d) Detection-discrimination dissociation at the critical presentation time of $8[8] \mathrm{ms}$. Although discrimination between upright and inverted faces did not differ significantly from chance, upright faces were localized and detected better than inverted faces. Error bars represent $95 \% \mathrm{Cls},{ }^{* * *} p<.001$. (e) Mean localization accuracy for upright and inverted faces for trials with correct (left panel) and incorrect (right panel) discrimination between upright and inverted faces. Inversion effects in localization did not depend on correct discrimination. Error bars represent $95 \% \mathrm{Cls}$ for the respective comparison between upright and inverted faces.

\section{Category-based attention requires awareness}

In Experiment 3 we demonstrated that the visual system is differentially sensitive to upright and inverted faces without the participant being consciously aware of this stimulus manipulation. In Experiment 4 we used the same detection-discrimination dissociation approach to test the (un)conscious origin of another previously reported detection difference: between objects that are preceded by a corresponding versus a non-corresponding verbal label ${ }^{37,38,44,45}$. When such word cues validly predict the category of the object, they are thought to improve detection through category-based attention ${ }^{76}$. Unlike face inversion, this effect involves a change in the observer's internal set rather than a stimulus manipulation. In b-CFS, suppression times are shorter when prior information (e.g. a word) predicts stimulus identity, category, or color ${ }^{37,38,44,45}$. We previously found such effects of category-based attention not only with CFS but also with other detection paradigms not involving interocular suppression ${ }^{37}$, indicating that the effect may, like the face inversion effect, reflect unconscious processing. However, in the absence of a measure to establish absence of consciousness, the effect could similarly reflect conscious modulation of stimulus processing ${ }^{77}$.

To distinguish between these accounts, in Experiment 4 we measured localization of object stimuli that were preceded by word cues providing valid or invalid information about the object's category (e.g. a picture of a cat preceded by the word "cat" or "cup", Figure 5a). Objects from eight different categories were presented for different presentation times ranging from 8 to $58 \mathrm{~ms}$ and backward masked. Importantly, we assessed participant's awareness of the critical manipulation underlying the effect. In addition to a 2-AFC localization task, participants also indicated on every trial whether the object was validly or invalidly cued (the order of the two tasks was counterbalanced between participants).

We ran two versions of the experiment that differed only with regard to the exact duration of the shortest and longest presentation time. Averaged across all presentation times validly cued objects were localized 
better than invalidly cued objects (Experiment $4 \mathrm{a}, F(1,44)=32.45, p<.001, \eta_{p}^{2}=.42, \mathrm{BF}_{10}=60.88$; Experiment $4 \mathrm{~b}, F(1,49)=20.50, p<.001, \eta_{p}{ }^{2}=.30, \mathrm{BF}_{10}=6.49$; Figure $5 \mathrm{~b}$, for subject-level display of localization and discrimination sensitivity, refer to Extended Data Figure 4), replicating previous findings $\mathrm{s}^{37}$. Cueing effects tended to be larger at intermediate presentation times than at the shortest and longest presentation times (Figure 5c). In Experiment 4a, at the shortest presentation time (8 ms, followed by a blank of $8 \mathrm{~ms}$ ) the cueing effect was significant $\left(t(44)=2.95, p=.005, d_{z}=0.44, \mathrm{BF}_{10}=\right.$ 6.97); however, also cue discriminability exceeded chance performance $(t(44)=2.08, p=.022$, onetailed, $d_{z}=0.31, \mathrm{BF}_{0+}=0.45$; Figure $5 \mathrm{c}$ and $5 \mathrm{~d}$ ), such that it was not possible to determine whether the cueing effect occurred consciously or unconsciously. In Experiment 4b, we therefore reduced bottomup stimulus strength further and removed the 8-ms blank between the object and the mask for the shortest presentation time. In this condition, cue discriminability did not differ significantly from chance $\left(t(49)=-0.35, p=.36\right.$, one-tailed, $\left.d_{z}=0.05, \mathrm{BF}_{0+}=8.36\right)$, while localization sensitivity was well-above chance for both validly and invalidly cued objects (both $t(49)>6.68, p<.001, d_{z}>0.94, \mathrm{BF}_{10}>6.30 \times 10^{5}$; Figure 5d). Critically, however, there was no significant difference in localization sensitivity between validly and invalidly cued objects $\left(t(49)=0.36, p=.72, d_{z}=0.05, \mathrm{BF}_{01}=6.11\right)$. Thus, only when observers had some ability to discriminate the validity of the cue did the cue influence object localization, indicating that the effect of category-based attention requires conscious object processing.

To provide additional evidence for a tight link between the category-based cueing effect and awareness, we compared trials with correct and incorrect discrimination responses. If the cueing effect did indeed depend on the ability to discriminate cue validity, it should be larger in trials with correct than with incorrect discrimination responses. We tested this in participants who had enough data points with correct and indirect discrimination responses (Experiment 4a: 37/45 participants, Experiment 4b: 47/50 participants, excluding the longest presentation times with ceiling discrimination performance, see Methods). Supporting our prediction, the localization cueing effect was highly significant for trials in which cue discrimination was correct (Experiment $4 \mathrm{a}, F(1,36)=64.29, p<.001, \eta_{p}{ }^{2}=.64, \mathrm{BF}_{10}=$ $1.51 \times 10^{10}$; Experiment $4 b, F(1,46)=20.29, p<.001 \eta_{p}^{2}=.31, \mathrm{BF}_{10}=910.97$; Figure 5e), but not when cue discrimination was incorrect (the effect even tended to reverse: Experiment $4 \mathrm{a}, F(1,36)=6.99, p=$ $.012, \eta_{p}^{2}=.16, \mathrm{BF}_{10}=3.25$; Experiment $4 \mathrm{~b}, F(1,46)=3.11, p=.085, \eta_{p}^{2}=.06$, but $\mathrm{BF}_{10}=0.66$ ); this difference was reflected in significant interactions between cue validity and discrimination accuracy (Experiment $4 \mathrm{a}, F(1,36)=33.77, p<.001, \eta_{p}^{2}=.48, \mathrm{BF}_{10}=3.51 \times 10^{8}$; Experiment $4 \mathrm{~b}, F(1,46)=16.14, p$ $\left.<.001, \eta_{p}^{2}=.26, \mathrm{BF}_{10}=1.81 \times 10^{3}\right)$.

Together, these findings indicate that the detection advantage of validly cued objects depends on awareness; therefore, this form of category-based attention does not affect unconscious processing. 
a

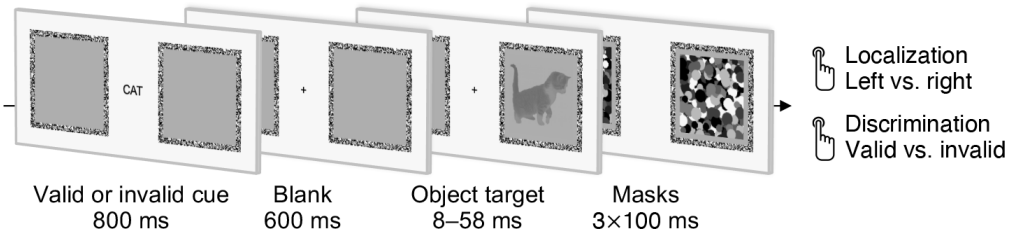

b
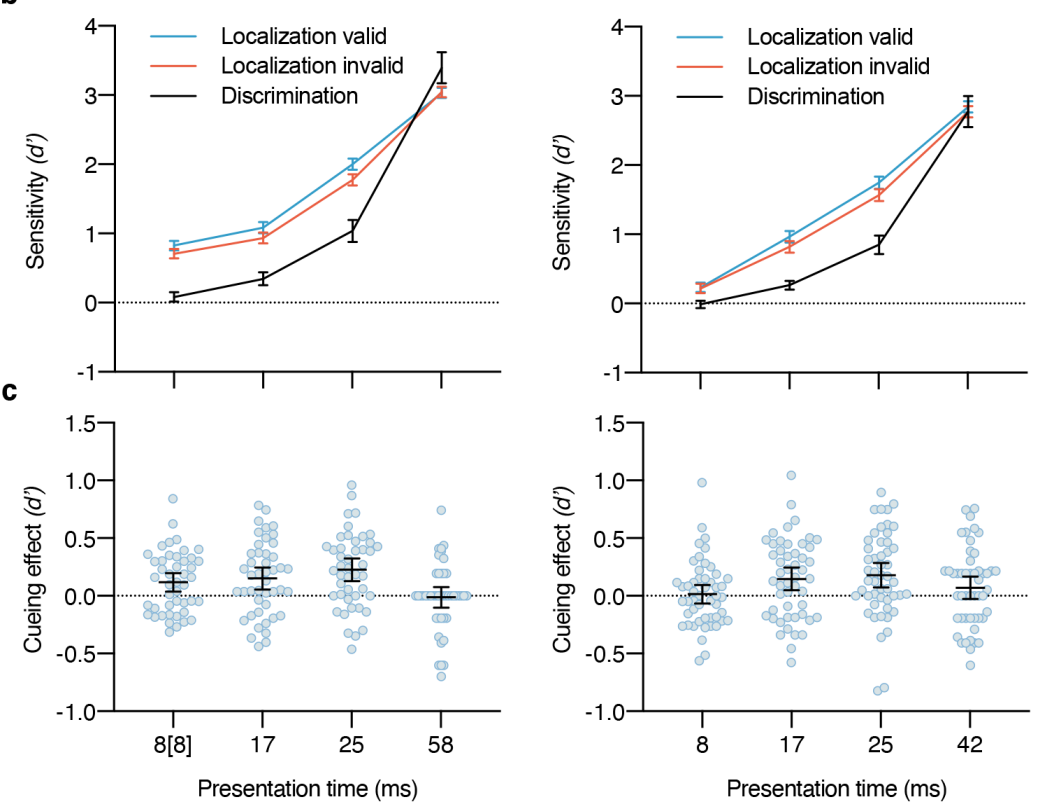

d

Presentation $8[8]$ vs. $8 \mathrm{~ms} \quad \mathrm{e}$

Discrimination correct

Discrimination incorrect
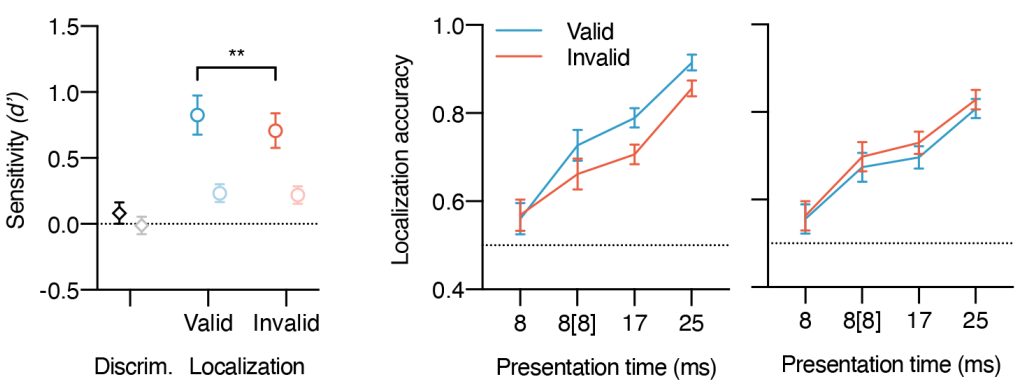

Figure 5. Experiment 4, testing the influence of category-based attention on conscious vs. unconscious

processing. (a) At the beginning of a trial a word provided either valid or invalid information about the category membership of the object target that was subsequently presented for four different fixed presentation times and backward masked. On every trial, participants performed two tasks (order counterbalanced between participants) measuring object localization (2-AFC) and discrimination of valid vs. invalid cues. (b) Mean localization sensitivity for validly and invalidly cued objects for the four different presentation times (values in square brackets refer to an additional blank screen of $8 \mathrm{~ms}$ between the face stimulus and the mask) in Experiment $4 \mathrm{a}$ (left panel) and in Experiment $4 \mathrm{~b}$ (right panel). The two versions of the experiment differed with regard to the shortest and longest presentation time. Error bars represent $95 \%$ $\mathrm{Cls}$ for the respective comparison between valid and invalid cues. For comparison, both panels also show mean sensitivity and $95 \% \mathrm{Cls}$ for discriminating between valid and invalid cues for every presentation time (c) Cueing effects ( $d$ 'in valid minus invalid trials) for every presentation time. Circles represent individual participants (left 
panel, Experiment $4 a, N=45$; right panel, Experiment $4 b, N=50$ ), horizontal lines the means, and error bars 95\% Cls. (d) No evidence for a detection-discrimination dissociation at the critical presentation times of 8[8] $\mathrm{ms}$ (saturated symbols) and $8 \mathrm{~ms}$ (light symbols). At $8[8] \mathrm{ms}$, the cueing effect was significant $\left({ }^{* *} p<.01\right.$ ) but discrimination between valid and invalid cues was significantly above chance $(p<.05)$. At $8 \mathrm{~ms}$, discrimination did not differ significantly from chance but there was no significant cueing effect either. Error bars represent 95\% Cls. (e) Mean localization accuracy for validly and invalidly cued objects for trials with correct (left panel) and incorrect (right panel) discrimination between valid and invalid cues (collapsed across Experiment 4a and 4b). Cueing improved localization only in trials with correct discrimination responses. Error bars represent $95 \%$ $\mathrm{Cls}$ for the respective comparison between valid and invalid cues.

\section{Discussion}

Evidence from visual detection paradigms suggests that many of the functions commonly associated with consciousness can occur unconsciously $y^{7,16,21}$. However, because detection differences can result from both conscious and unconscious processes, these need to be dissociated experimentally. Here, we showed that the popular breaking CFS paradigm cannot dissociate conscious and unconscious contributions to detection effects. As a solution, we presented a paradigm that combines a detection and a discrimination task and demonstrated how it can be used to distinguish between functions that do and do not require consciousness.

In the b-CFS paradigm, seemingly CFS-specific effects could reflect the insensitivity of control conditions rather than unconscious processing under interocular suppression. With optimally designed control paradigms, we obtained effects similar to CFS. Although these different paradigms involve partially distinct neural mechanisms, a difference in detection may nevertheless involve shared mechanisms. For example, the detection advantage of upright over inverted faces may involve firing of face-selective cells in a rapid feedforward sweep through the visual pathways ${ }^{78}$. Masking and inattention (RSVP) are thought to leave such feedforward processing intact ${ }^{63,79,80}$. Interocular suppression may interfere more strongly with early visual processing (e.g. in primary visual cortex) ${ }^{49,51,81}$, resulting in overall much longer periods of invisibility. However, when suppression weakens over time, a detection difference could nevertheless reflect similar mechanisms, for example activity in face-selective cells evoked by information that eventually escapes initially strong suppression.

But are these mechanisms unconscious or conscious? To answer this question, an additional task needs to establish absence of conscious processing ${ }^{66}$. Adopting the detection-discrimination dissociation paradigm presented here we found that upright faces were detected better than inverted faces even when participants could not distinguish between upright and inverted faces. These results demonstrate that 
face orientation can be processed unconsciously, consistent with the idea that face detection can be achieved by face-selective cells in a rapid, unconscious feedforward sweep ${ }^{78,82}$. As face inversion keeps physical stimulus properties intact but disrupts spatial relationships among facial features, the face inversion effect is often considered to be a marker of visual integration. As such, our findings have implications for current debates revolving around whether consciousness is required for integration of information ${ }^{50,51,83,84}$. Proponents of unconscious integration cite the face inversion effect in b-CFS as evidence for integration ${ }^{50}$, while sceptics question the validity of the b-CFS paradigm ${ }^{52}$. Our findings now provide clear evidence for an unconscious origin of the inversion effect. Future work adopting the present paradigm will determine whether unconscious integration is limited to highly familiar and overlearned stimuli such as faces or bodies ${ }^{47,85}$, and whether unconscious integration can happen across multiple objects ${ }^{86}$, different modalities ${ }^{8}$, and time ${ }^{10}$.

Another debate with important theoretical implications concerns the relationship between consciousness and attention ${ }^{9,63,87}$. Preparatory top-down attention evokes anticipatory activity in sensory areas in a content-specific fashion, enhancing neural processing and detection of stimuli matching the attended dimension ${ }^{76,88}$. Consistent with the view that such attentional enhancement is tightly linked to conscious awareness ${ }^{89-91}$, we found that the effect of category-based attention required at least some conscious access to the validity of the cue. This may seem at odds with recent studies suggesting that some forms of attention can be dissociated from consciousness ${ }^{9,87}$. However, these studies did not always convincingly rule out conscious awareness (also see next paragraph). Furthermore, these studies manipulated overall processing resources (load) $)^{92-95}$, spatial attention ${ }^{96,97}$, or feature-based attention ${ }^{98}$ rather than category-based attention. Spatial attention may act as a filter at early, unconscious levels, independent of stimulus content. Similarly, for simple stimulus features such as colour and orientation, attention may enhance feedforward processing in early visual cortex ${ }^{99}$. Attentional enhancement in highlevel visual cortex representing the shape and category of the cued objects, by contrast, appears to require awareness. This finding is consistent with evidence from human and monkey electrophysiology showing that attention to such more complex stimulus features modulates visual cortex responses relatively late in time ${ }^{100,101}$, likely reflecting local recurrent processing or feedback from downstream areas such as prefrontal cortex ${ }^{102}$. These processes may inevitably give rise to awareness ${ }^{77,87}$. By showing that categorybased attention cannot be dissociated from consciousness, our findings lend support to theories in which the control of attention is a key function of consciousness, such as global workspace theory ${ }^{3,5,63}$ or attention schema theory ${ }^{103}$.

The current findings highlight how the detection-discrimination dissociation paradigm can distinguish between conscious and unconscious influences on behaviour, satisfying the most rigorous criteria for 
demonstrating unconscious processing while addressing central objections that have been raised against other approaches. Perhaps most importantly, studies on unconscious processing are often criticized for not convincingly establishing absence of consciousness ${ }^{13-15}$. Awareness measures do not always capture the information driving the unconscious effect ${ }^{66,67}$, they may be susceptible to criterion biases ${ }^{104}$, or they are collected in separate blocks with fewer trials or fewer participants, resulting in insufficient power ${ }^{105,106}$. In the present paradigm, the discrimination task represents an exhaustive measure of awareness specifically designed to capture the information relevant for the detection effect. Performance in this task was measured in the same trials as detection, using a criterion-free measure of sensitivity. By finding a presentation condition with zero discriminability across the whole group, no participants or trials needed to be excluded based on the awareness measure; such data exclusion is a common procedure that risks severely inflating estimates of unconscious processing due to regression to the mean ${ }^{107}$. Furthermore, while absence of awareness is often demonstrated by (inappropriately) accepting the null hypothesis when the awareness measure is not statistically significant, we used Bayesian statistics to provide evidence in support of null sensitivity ${ }^{108}$. Finally, the detection effect can be directly compared to the measure of awareness on the same scale ${ }^{66}$, a statistical comparison that is almost never performed although it is key for claiming unconscious processing ${ }^{75}$.

The detection-discrimination dissociation approach can distinguish between conscious and unconscious contributions to detection. A detection difference alone, obtained with CFS, backward masking, or other methods for stimulus presentation, may not reflect unconscious processing. Indeed, opposite results for the face inversion effect and the effect of category-based attention demonstrate that not all detection differences reflect unconscious processing. Accordingly, we suspect that some of the striking effects obtained with detection paradigms such as b-CFS reflect genuinely unconscious processes, while others will turn out to arise from conscious processes. In a thriving research field zeroing in on the functions and adaptive advantage of consciousness, this paradigm will help to map out the scope and limits of unconscious processing. If our conclusions about the tight link between consciousness and category-based attention are right, this research program may reveal that consciousness is more than an epiphenomenon, assigning consciousness an important place in the neurocognitive architecture of the human mind. 


\section{Methods}

\section{Participants}

All procedures were approved by the local ethics committees. Participants gave informed consent and received either course credit or a small monetary compensation for their participation. A total of 305 participants took part in the experiments. In Experiment 1, there were 24 participants (19 female, mean age 21.4 years, $S D=3.4$ ) who all completed the dichoptic-masking paradigm and then the monopticmasking paradigm in one session, and the CFS paradigm and the simultaneous-noise masking paradigm in another session on a different day. With the exception of one author of the paper and one student involved in data collection, all participants were recruited through the University of Trento participant pool and were naïve to the purpose of the study. For all other experiments, participants were recruited through the University of Amsterdam participant pool and all were naïve to the purpose of the study. In Experiment 2, eight participants were excluded from the CFS experiment because their overall localization performance across all presentation times was at floor (six participants, accuracy less than $51 \%$ correct) or at ceiling (two participants, accuracy above 99\% correct), and one participant was excluded from the masking and RSVP experiment because of low accuracy for the first target in the RSVP. The final samples consisted of 40 participants in the CFS experiment (32 female, mean age 23.8 years, $S D=8.5$ ), and another 40 participants ( 18 female, mean age 22.6 years, $S D=3.3$ ) who took part in both the masking and the RSVP experiment in the same session (order counterbalanced between participant). In Experiment 3, data from four participants was excluded because either their overall localization or discrimination performance was below 55\% correct at the longest presentation times, resulting in a final sample of 94 participants (61 female, mean age 22.6 years, $S D=7.0$ ). In Experiment 4, 45 participants completed Experiment 4a (36 female, mean age 20.1 years, $S D=3.2$ ), and 50 participants Experiment $4 \mathrm{~b}$ (42 female, mean age 19.8 years, $S D=2.0$ ). All participants reported normal or correct-to-normal vision. In all experiments, participants received detailed written and verbal instructions and practice trials, and all experiments contained several short obligatory breaks. Participants were instructed to respond as accurately as possible, without speed pressure.

\section{Sample sizes and statistical power}

Power calculations were carried out in Matlab (using the Statistics toolbox function "sampsizepwr") for paired $t$-tests (effect size measure $d_{z}$ ) Given the substantial size of previously reported b-CFS effects, we decided to test 24 participants in Experiment 1 (resulting in 80\% power to detect $d_{z}>0.60$ ). To provide more evidence for effects of similar size with other paradigms, 40 participants were tested with each paradigm in Experiment 2 (80\% power to detect $\left.d_{z}>0.46\right)$. For Experiment 3, to probe whether detection-discrimination dissociations exist and to get a more precise estimate of the size of effect, the goal was to test a maximally large number of participants in the project period (which resulted in $80 \%$ 
power to detect $d_{z}>0.30$ ). For Experiment $4 \mathrm{a}$ and $4 \mathrm{~b}$, the sample size was based on the inversion effect size in Experiment $3\left(d_{z}=0.42\right)$; data collection ended at the end of the project period (resulting in 78\% and $82 \%$ power to detect $d_{z}=0.42$ in Experiment $4 \mathrm{a}$ and $4 \mathrm{~b}$, respectively).

\section{Display and apparatus}

In Experiment 1, stimuli were presented on a 21 -inch CRT monitor $(1024 \times 768$ pixels resolution, 160 $\mathrm{Hz}$ refresh rate). In the other experiments, stimuli were presented on 24-inch LCD monitors (all with $1920 \times 1080$ pixels resolution, $100 \mathrm{~Hz}$ refresh rate in Experiment 2, $120 \mathrm{~Hz}$ refresh rate in Experiment 3 and 4). Screens were gamma-corrected and presentation times were synchronized with the vertical refresh cycles of the screens. In Experiment 1 for all four paradigms and in Experiment 2 for the CFS paradigm, participants viewed the screen dichoptically through a custom-built mirror stereoscope using a chin-and-head rest placed approximately $60 \mathrm{~cm}$ in front of the screen. The mirrors of the stereoscope were adjusted for each observer to yield stable binocular fusion. For all other experiments and paradigms, participants viewed the screen binocularly from a free viewing distance of approximately 60 $\mathrm{cm}$. Experiments were programmed in Matlab using Psychtoolbox ${ }^{109}$ functions.

In Experiments 1-3, stimuli were presented in a central grey box (experiment 1: $10.1^{\circ} \times 10.1^{\circ}$ of visual angle; experiment 2 and $3: 7.5^{\circ} \times 7.5^{\circ}$ ) with (fusion) contours (width $0.2-0.3^{\circ}$ ) consisting of random black and white pixels. The remainder of the screen was black. In Experiment 1 and in the CFS part of Experiment 2, two boxes were displayed side-by-side on the screen such that one contour was shown to each eye (distance between the centres of the two boxes, Experiment 1: $21.0^{\circ}$, Experiment 2: $15.0^{\circ}$ ). In the centre of each contour a small white fixation cross was displayed. In Experiment 4, stimuli were presented in one of two boxes $\left(4.1^{\circ} \times 4.1^{\circ}\right)$ that were cantered left and right of central fixation (distance to the centre of each box $4.2^{\circ}$ ). Participants were asked to fixate during the experimental trials. In all experiments, trials started with a 1-s fixation period in which only the fusion contours and the fixation cross were presented (in Experiments 2-4 the fixation cross then disappeared for $0.5 \mathrm{~s}$ to mark the start of the stimulus presentation sequence).

\section{Stimuli}

Face stimuli were 40 emotionally-neutral face photographs taken from the Karolinska face data set. We removed hair and outer facial features, converted the stimuli to greyscale, and resized the stimuli to fit within a square image (Experiment 1: $4.0^{\circ} \times 4.0^{\circ}$, Experiment 2 and 3: $2.6^{\circ} \times 2.6^{\circ}$ ) of the same grey background as the rest of the central box for stimulus presentation. The mean luminance and the contrast within the area corresponding to the face was equated (RMS contrast, Experiment 1 low contrast stimuli: 0.07, Experiment 1 high contrast stimuli: 0.22; Experiment 2 and 3: 0.13), and the outer 
face contour was blurred into the background. These images were presented either upright or inverted (i.e., rotated by 180 degrees). In Experiment 4 stimuli were 48 greyscale photographs of objects from eight categories (cars, cats, chairs, cups, guitars, hammers, persons, and shoes). For each category, six different exemplars were included, seen from different viewing angles. Objects were fit to a square image $\left(3.2^{\circ} \times 3.2^{\circ}\right)$ of the same grey background as the boxes for stimulus presentation and mean luminance and contrast of the area corresponding to the object were equated (RMS contrast 0.15 ).

For the CFS and backward masking paradigms, we generated 100 greyscale masks (Experiment 1: 9.5 $\times 9.5^{\circ}$, Experiment 2 and 3: $7.5^{\circ} \times 7.5^{\circ}$, Experiment 4: $3.7^{\circ} \times 3.7^{\circ}$ ) consisting of randomly arranged circles (diameter $0.3-1.5^{\circ}$ ). For the simultaneous noise masking paradigm, for every face exemplar we created 100 corresponding greyscale noise backgrounds. These noise backgrounds $\left(9.5^{\circ} \times 9.5^{\circ}\right)$ consisted of six phase-scrambled versions of the respective square image that were spatially concatenated and cut to fit into the fusion contour. For the RSVP paradigm, 352 scrambled distracters were generated by dividing the inner part of the (upright and inverted) face stimuli into grids consisting of 16 or 20 rectangles of different sizes and randomly rearranging these rectangles. As a first attention-demanding target (T1) in the RSVP, we created 80 stimuli that consisted either of a random arrangement of differently sized circles or diamonds. These were then cut to correspond to the contours of the (upright and inverted) face stimuli, and displayed through the green RGB channel only (rendering T1 salient in the stream of grey distracters).

\section{Experiment 1}

The exact way of stimulus presentation differed between paradigms, but in all paradigms upright or inverted faces were presented either above, below, left or right of the fixation cross (centre-to-centre distance $2.6^{\circ}$ ), and participants performed a 4-AFC localization task. Following an incorrect response, presentation time was increased; following three consecutive correct responses, presentation was decreased (1-up, 3-down). The specific staircase parameters in each paradigm were based on pilot studies. At the end of the stimulus presentation sequence participants pressed one of the four arrow keys on the keyboard to indicate in which of the four possible locations the face stimulus was presented. Afterwards, they indicated their confidence in this response on a scale from 1-4 (confidence scores are not reported here).

In CFS, the face stimulus was gradually faded in to one eye by decreasing its transparency from $100 \%$ to $0 \%$ over the first $400 \mathrm{~ms}$ of a trial. A random sequence of masks changing every $100 \mathrm{~ms}$ was presented to the other eye. The contrast of the masks was decreased linearly to zero over $10 \mathrm{~s}$. Presentation time of the face stimuli was adjusted by staircases, which started with a presentation time of $3.6 \mathrm{~s}$, and had a step 
size of $400 \mathrm{~ms}$, a minimum value of $400 \mathrm{~ms}$, and a maximum value of $10 \mathrm{~s}$. At the end of the stimulus sequence, three masks were presented for $100 \mathrm{~ms}$ each to both eyes to prevent afterimages. In simultaneous noise masking, both the face stimulus and a random sequence of noise masks changing every $100 \mathrm{~ms}$ were presented to the same eye. The other eye was presented with the frame only. The face was gradually faded in by decreasing its transparency from $100 \%$ to $0 \%$ over the first $2.4 \mathrm{~s}$ of a trial. Staircases started with a presentation time of $3.6 \mathrm{~s}$, and had a step size of $100 \mathrm{~ms}$, a minimum value of $100 \mathrm{~ms}$, and a maximum value of $8 \mathrm{~s}$. In monoptic backward masking, the face stimulus was presented briefly to one eye, followed directly by three randomly selected masks (100 ms each) to the same eye. Staircases started with a presentation time of $75 \mathrm{~ms}$, and had a step size of $6.25 \mathrm{~ms}$, a minimum value of $6.25 \mathrm{~ms}$, and a maximum value of $625 \mathrm{~ms}$. In dichoptic backward masking, the parameters were the same as in monoptic backward masking, except that the face and the masks were presented to different eyes.

The experimental design was the same for all four paradigms. There were 640 trials, in which each combination of face orientation (upright, inverted), face contrast (low, high), and eye for face presentation occurred 80 times. Eight interleaved staircases controlled stimulus presentation times for these eight conditions. Each of the four stimulus locations occurred equally often in each condition, and each of the 40 face exemplars was shown equally often. Trial order was randomized.

For every staircase, a separate threshold was calculated as the mean of the last five reversals. Thresholds for the four conditions of interest (upright/inverted $\times$ low-/ high-contrast) were then calculated by averaging over the values from both eyes. We were not interested in the interaction between face orientation and face contrast, and thus averaged over the factor of no interest. We also calculated effects from transformed data, following the recommended latency-normalization procedure ${ }^{72}$ where for each participant the contrast/inversion effect (calculated from mean localization thresholds) was divided by the overall localization threshold, such that it reflects a proportional difference in localization thresholds. For standardized mean difference effect sizes $d_{z}$ we calculated 95\% CIs (Figure 1d) using the MBESS package $^{110,111}$ for $\mathrm{R}$.

\section{Experiment 2}

CFS and masking were similar to Experiment 1 but in Experiment 2 presentation times were fixed. Participants performed a 2-AFC localization task on upright or inverted faces that were presented either left or right of the central fixation cross (centre-to-centre distance $2.6^{\circ}$ ). In the rapid serial visual presentation (RSVP) paradigm, participants additionally reported the identity of a T1 stimulus (circles vs. diamonds, using the " 1 " and " 2 " keys), before indicating face location with one of the two arrow keys. Afterwards, they reported subjective awareness on the PAS or on a continuous scale (not reported here). 
In CFS, the face stimulus was faded in by decreasing its transparency from $100 \%$ to $0 \%$ over the first 200 ms of a trial. Face stimuli were presented for either 200, 400, or $800 \mathrm{~ms}$ to one eye, while a random sequence of masks changing every 100 ms was presented to the other eye. Stimulus presentation was followed by three masks presented to both eyes to prevent afterimages. To determine the eye for face presentation, in an initial calibration block of 48 trials participants localized scrambled faces (the distracters from the RSVP paradigm) that were presented for $400 \mathrm{~ms}$ to the participant's dominant eye (as determined with the hole-in-the-card test ${ }^{112}$ ). If accuracy exceeded $70 \%$ in this block, in the experiment proper face stimuli were presented to the participant's non-dominant eye; otherwise to the dominant eye. This was done to avoid ceiling performance. Pilot testing and previous studies ${ }^{70,113,114}$ indicated that CFS weakens over time, and switching stimulus presentation to the non-dominant eye should result in overall longer suppression time ${ }^{115}$. In standard backward masking, a face stimulus was presented for 10, 20, or $30 \mathrm{~ms}$ and followed directly by three randomly selected masks (100 ms each). In the RSVP paradigm, three different streams of 24 randomly selected scrambled distracters changing every $100 \mathrm{~ms}$ were presented in the left, right, and central location. After the presentation of seven to eleven distracters (selected at random), the "T1" stimulus (green circles or diamonds) was presented in the central stream; the face "T2" stimulus followed after one, two, or six distracters in the left or right stream (resulting in T1-T2 SOAs of 200, 300, and $700 \mathrm{~ms}$ ).

The experimental design was the same for all four paradigms. There were 384 trials, in which each combination of presentation time, face orientation, stimulus location, and 16 face exemplars occurred twice. Trial order was randomized. For the RSVP paradigm, instructions highlighted the importance of correctly reporting $\mathrm{T} 1$ to ensure attentional engagement. Trials with incorrect $\mathrm{T} 1$ responses $(M=3.5 \%$, $S D$ 3.3) were excluded from the analyses.

\section{Experiment 3}

In stimulus-present trials, an upright or inverted face stimulus was presented to the left or to the right of the centre of the box (centre-to-centre distance $\left.2.6^{\circ}\right)$. There were five presentation times $(8.3,8.3[8.3]$, 16.7, 25.0, and 33.3 [8.3] ms), two of which contained an additional presentation of the blank central box for $8.3 \mathrm{~ms}$ after the presentation of the face stimulus (values in square brackets). Three backward masks were presented for $100 \mathrm{~ms}$ each. In stimulus-absent trials face presentation was replaced with the blank central box. Presentation times were selected based on pilot testing. We included several longer presentation times that resulted in high sensitivity. This was done to map the whole psychometric function, to provide participants with a clear signal, and to keep participants on task and motivated (to avoid underestimating awareness). 
At the end of the stimulus presentation sequence participants completed three tasks. In the localization task, they indicated whether the face stimulus appeared to the left or to the right of the centre of the box, using the left/right arrow keys. In what we refer to as the detection task, they indicated subjective awareness on the Perceptual Awareness Scale (PAS) ${ }^{74}$, using the 1-4 buttons, corresponding to "no experience", "brief glimpse", "almost clear experience", and "clear experience". The PAS is a widely adopted scale that is often thought to optimally capture phenomenology of briefly presented, masked stimuli. Here, we converted PAS responses to a criterion-free measure of stimulus detectability ( $d$, see below). In the discrimination task, they indicated whether the face stimulus was presented in upright or in inverted orientation, using the up/down arrow keys. Response assignments for each task were displayed in the box. The order of the three tasks was counterbalanced between participants; due to a procedural error slightly different numbers of participants were assigned to the six possible task orders (range between 12 and 18 participants per condition).

There were 1,080 trials, in which each of the five presentation times occurred equally often. In one third of the trials no face was presented. In the remaining 720 trials, each combination of two target locations (left/right), two target orientations (upright/inverted), and 36 face exemplars occurred equally often for each presentation time. Trial order was randomized. Before starting the experiment, participants received detailed instructions, including a description of the PAS and its four levels, and extensive practice. This included a description of the experimental design (different presentation times, proportion of face-present and face-absent trials, examples of upright and inverted faces) and several short practice blocks.

Responses from all three tasks were transformed to the SDT measure $d^{\prime}$, separately for each presentation time. For localization, "left" responses were coded as hits in trials where the face was presented in the left location, and as false alarms in trials where the face was presented in the right location. For detection, "brief glimpse", "almost clear experience", and "clear experience" responses on the PAS were coded as hits in face-present trials (separately for upright and inverted faces) and as false alarms in face-absent trials. For the discrimination measure, "upright" responses were coded as hits in upright-face trials and as false alarms in inverted-face trials. For all three measures, hit and false alarm rates of 0 or 1 were converted to $1 /(2 N)$ and $1-1 /(2 N)$, respectively, with $N$ being the number of trials on which the rates were based ${ }^{116}$. The $z$-transformed false alarm rate was then subtracted from the $z$-transformed hit rate to yield $d$. To adjust for the fact that, in SDT terms, the localization task is a 2-AFC task while the detection and the discrimination task are yes/no tasks, for the localization task $d$ ' was divided by the square root of two ${ }^{116}$. 
To compare inversion effects in trials with correct and incorrect discrimination responses, we included participants who had at least five trials per condition (upright and inverted faces) with correct and incorrect discrimination response at the four shortest presentation times (excluding the longest presentation time where discrimination performance was at ceiling). For this analysis we calculated the proportion of correct localization responses. In the 2-AFC localization task, accuracy represents another criterion-free index of sensitivity, but is less affected by the low number of trials than $d$. In the Supplementary Information we report additional analyses of trials with correct and incorrect discrimination responses.

\section{Experiment 4}

At the beginning of a trial a word cue denoting one of the eight object categories was presented for 800 ms in white uppercase Arial font in the centre between the two boxes. In valid trials (50\% of trials), the word corresponded to the category of the upcoming object stimulus. In invalid trials ( $50 \%$ of trials), the word was randomly selected from the seven other categories. The cue was followed by a $600 \mathrm{~ms}-$ presentation of the empty boxes only. An object stimulus was then presented for one of four presentation times (Experiment 4a: 8.3[8.3], 16.7, 25.0, or $58.3 \mathrm{~ms}$, Experiment 4b: 8.3, 16.7, 25.0, or $41.7 \mathrm{~ms}$ ) in the left or right box (distance from fixation to centre $4.2^{\circ}$ ), followed by three backward masks in each box that were presented for $100 \mathrm{~ms}$ each. Presentation times were selected based on pilot testing. Except for the shortest and longest presentation times Experiment $4 \mathrm{a}$ and $4 \mathrm{~b}$ were identical.

Participants completed two tasks on every trial. In the localization task, they indicated whether the object stimulus appeared in the left or in the right box, using the left/right arrow keys. In the discrimination task, they indicated whether the cue validly or invalidly predicted the category of the object stimulus, using the up/down arrow keys on the keyboard. Response assignments for each task were displayed in the box. The order of the two tasks was counterbalanced between participants.

The experiment consisted of 768 trials, in which each combination of presentation time, target location, cue validity, eight object categories, and six object exemplars occurred once. Trial order was randomized. Participants received detailed instructions, including a description of the experimental design (different presentation times, eight object categories, examples of valid and invalid cues) and several short practice blocks. Before starting the experiment, participants had to achieve a criterion of at least $90 \%$ correct in the discrimination task in a block of ten trials with a presentation time of $66.7 \mathrm{~ms}$. Participants were informed that there were $50 \%$ valid and 50\% invalid trials, and they were asked to pay attention to (i.e., to read) the cues and try to use them to perform well. Responses from the two tasks were transformed 
to $d$, following the description for Experiment 3 above. Analyses of cueing effects in trials with correct and incorrect discrimination responses were analogous to the description of Experiment 3 above (see the Supplementary Information, Supplementary Table 1, and Supplementary Table 2 for additional analyses).

\section{Statistics}

Data distribution was assumed to be normal but this was not formally tested. We report both standard frequentist statistics and Bayes factors (BFs) calculated in JASP ${ }^{117}$ with default prior scales. When frequentist statistics indicate a significant effect, the corresponding BF is reported as a quantification of the evidence for the alternative hypothesis $\left(\mathrm{BF}_{10}\right)$; when the effect is not significant, the reported $\mathrm{BF}$ quantifies the evidence for the null hypothesis $\left(\mathrm{BF}_{01}\right)$. To demonstrate absence of awareness in the discrimination tasks of Experiment 3 and 4, the directional $\mathrm{BF}_{0+}$ quantifies evidence for null sensitivity compared to the alternative of above-chance performance. For multi-factorial ANOVAs, we report the inclusion BF quantifying the evidence for all models containing a particular effect compared to all models without that effect.

\section{Data availability}

Data are available at https://osf.io/sn8cr/.

\section{Code availability}

Custom code that supports the findings of this study is available from the corresponding author upon request.

\section{References}

1. Cohen, M. A. \& Dennett, D. C. Consciousness cannot be separated from function. Trends Cogn. Sci. 15, 358-364 (2011).

2. Baars, B. J. Global workspace theory of consciousness: Toward a cognitive neuroscience of human experience. Prog. Brain Res. 150, 45-53 (2005).

3. Dehaene, S. \& Naccache, L. Towards a cognitive neuroscience of consciousness: Basic evidence and a workspace framework. Cognition 79, 1-37 (2001).

4. Kanai, R. et al. Information generation as a functional basis of consciousness. Neurosci. Conscious. 2019, niz016 (2019). 
5. Baars, B. J. A cognitive theory of consciousness. (Cambridge University Press, 1988).

6. Kanwisher, N. Neural events and perceptual awareness. Cognition 79, 89-113 (2001).

7. Hassin, R. R. Yes it can: On the functional abilities of the human unconscious. Perspect. Psychol. Sci. 8, 195-207 (2013).

8. Mudrik, L., Faivre, N. \& Koch, C. Information integration without awareness. Trends Cogn. Sci. 18, 488-496 (2014).

9. Koch, C. \& Tsuchiya, N. Attention and consciousness: Two distinct brain processes. Trends Cogn. Sci. 11, 16-22 (2007).

10. Soto, D. \& Silvanto, J. Reappraising the relationship between working memory and conscious awareness. Trends Cogn. Sci. 18, 520-525 (2014).

11. Van Gaal, S. \& Lamme, V. A. F. Unconscious high-level information processing: Implication for neurobiological theories of consciousness. Neuroscientist 18, 287-301 (2012).

12. Hesselmann, G. \& Moors, P. Definitely maybe: can unconscious processes perform the same functions as conscious processes? Front. Psychol. 6, 1-5 (2015).

13. Schmidt, T. Invisible stimuli, implicit thresholds: Why invisibility judgments cannot be interpreted in isolation. Adv. Cogn. Psychol. 11, 31-41 (2015).

14. Newell, B. R. \& Shanks, D. R. Unconscious influences on decision making: A critical review. Behav. Brain Sci. 37, 1-19 (2014).

15. Holender, D. Semantic activation without conscious identification in dichotic listening, parafoveal vision, and visual masking: A survey and appraisal. Behav. Brain Sci. 9, 1-23 (1986).

16. Gayet, S., Van Der Stigchel, S. \& Paffen, C. L. E. Breaking continuous flash suppression: Competing for consciousness on the pre-semantic battlefield. Front. Psychol. 5, 1-10 (2014).

17. Yang, E., Brascamp, J., Kang, M. S. \& Blake, R. On the use of continuous flash suppression for the study of visual processing outside of awareness. Front. Psychol. 5, 1-17 (2014).

18. Jiang, Y., Costello, P. \& He, S. Processing of invisible stimuli: Advantage of upright faces and recognizable words in overcoming interocular suppression. Psychol. Sci. 18, 349-355 (2007).

19. Stein, T. \& Sterzer, P. Unconscious processing under interocular suppression: Getting the right measure. Front. Psychol. 5, 1-5 (2014).

20. Stein, T., Hebart, M. N. \& Sterzer, P. Breaking continuous flash suppression: A new measure of unconscious processing during interocular suppression? Front. Hum. Neurosci. 5, 1-17 (2011).

21. Stein, T. The breaking continuous flash suppression paradigm: Review, evaluation, and outlook. in Transitions Between Consciousness and Unconsciousness (ed. Hesselmann, G.) 1-38 (Routledge, 2019).

22. Tsuchiya, N. \& Koch, C. Continuous flash suppression reduces negative afterimages. Nat. Neurosci. 8, 1096-1101 (2005). 
23. Axelrod, V., Bar, M. \& Rees, G. Exploring the unconscious using faces. Trends Cogn. Sci. 19, P35-45 (2015).

24. Abir, Y., Sklar, A. Y., Dotsch, R., Todorov, A. \& Hassin, R. R. The determinants of consciousness of human faces. Nat. Hum. Behav. 2, 194-199 (2018).

25. Stewart, L. H. et al. Unconscious evaluation of faces on social dimensions. J. Exp. Psychol. Gen. 141, 715-727 (2012).

26. Schmack, K., Burk, J., Haynes, J. D. \& Sterzer, P. Predicting subjective affective salience from cortical responses to invisible object stimuli. Cereb. Cortex 26, 3453-3460 (2016).

27. Gayet, S., Paffen, C. L. E., Belopolsky, A. V., Theeuwes, J. \& Van der Stigchel, S. Visual input signaling threat gains preferential access to awareness in a breaking continuous flash suppression paradigm. Cognition 149, 77-83 (2016).

28. Yang, E., Zald, D. H. \& Blake, R. Fearful expressions gain preferential access to awareness during continuous flash suppression. Emotion 7, 882-886 (2007).

29. Wang, L., Weng, X. \& He, S. Perceptual grouping without awareness: Superiority of Kanizsa triangle in breaking interocular suppression. PLoS One 7, 1-6 (2012).

30. Moors, P., Wagemans, J. \& De-Wit, L. Causal events enter awareness faster than non-causal events. PeerJ 2017, e2932 (2017).

31. Hung, S. M., Styles, S. J. \& Hsieh, P. J. Can a word sound like a shape before you have seen it? Sound-shape mapping prior to conscious awareness. Psychol. Sci. 28, 263-275 (2017).

32. Stein, T., Kaiser, D. \& Peelen, M. V. Interobject grouping facilitates visual awareness. J. Vis. 15, $1-11(2015)$.

33. Alsius, A. \& Munhall, K. G. Detection of audiovisual speech correspondences without visual awareness. Psychol. Sci. 24, 423-431 (2013).

34. Tan, J. S. \& Yeh, S. L. Audiovisual integration facilitates unconscious visual scene processing. J. Exp. Psychol. Hum. Percept. Perform. 41, 1325-1335 (2015).

35. Zhou, W., Jiang, Y., He, S. \& Chen, D. Olfaction modulates visual perception in binocular rivalry. Curr. Biol. 20, 1356-1358 (2010).

36. Zhang, P., Jiang, Y. \& He, S. Voluntary attention modulates processing of eye-specific visual information. Psychol. Sci. 23, 254-260 (2012).

37. Stein, T. \& Peelen, M. V. Content-specific expectations enhance stimulus detectability by increasing perceptual sensitivity. J. Exp. Psychol. Gen. 144, 1089-1104 (2015).

38. Pinto, Y., van Gaal, S., de Lange, F. P., Lamme, V. A. F. \& Seth, A. K. Expectations accelerate entry of visual stimuli into awareness. J. Vis. 15, 1-15 (2015).

39. Gayet, S., Paffen, C. L. E. \& Van der Stigchel, S. Information matching the content of visual working memory is prioritized for conscious a ccess. Psychol. Sci. 24, 2472-2480 (2013). 
40. Pan, Y., Lin, B., Zhao, Y. \& Soto, D. Working memory biasing of visual perception without awareness. Attention, Perception, Psychophys. 76, 2051-2062 (2014).

41. Hung, S. M. \& Hsieh, P. J. Syntactic processing in the absence of awareness and semantics. J. Exp. Psychol. Hum. Percept. Perform. 41, 1376-1384 (2015).

42. Yang, Y. H. \& Yeh, S. L. Accessing the meaning of invisible words. Conscious. Cogn. 20, 223-233 (2011).

43. Costello, P., Jiang, Y., Baartman, B., McGlennen, K. \& He, S. Semantic and subword priming during binocular suppression. Conscious. Cogn. 18, 375-382 (2009).

44. Lupyan, G. \& Ward, E. J. Language can boost otherwise unseen objects into visual awareness. Proc. Natl. Acad. Sci. U. S. A. 110, 14196-14201 (2013).

45. Ostarek, M. \& Huettig, F. Spoken words can make the invisible visible-Testing the involvement of low-level visual representations in spoken word processing. J. Exp. Psychol. Hum. Percept. Perform. 43, 499-508 (2017).

46. Sklar, A. Y. et al. Reading and doing arithmetic nonconsciously. Proc. Natl. Acad. Sci. U. S. A. 109, 19614-19619 (2012).

47. Stein, T., Sterzer, P. \& Peelen, M. V. Privileged detection of conspecifics: Evidence from inversion effects during continuous flash suppression. Cognition 125, 64-79 (2012).

48. Zhou, G., Zhang, L., Liu, J., Yang, J. \& Qu, Z. Specificity of face processing without awareness. Conscious. Cogn. 19, 408-412 (2010).

49. Sterzer, P., Stein, T., Ludwig, K., Rothkirch, M. \& Hesselmann, G. Neural processing of visual information under interocular suppression: A critical review. Front. Psychol. 5, 453 (2014).

50. Sklar, A. Y., Deouell, L. Y. \& Hassin, R. R. Integration despite fractionation: Continuous flash suppression. Trends Cogn. Sci. 22, 956-957 (2018).

51. Moors, P., Hesselmann, G., Wagemans, J. \& van Ee, R. Continuous Flash Suppression: Stimulus Fractionation rather than Integration. Trends Cogn. Sci. 21, 719-721 (2017).

52. Moors, P. et al. Three criteria for evaluating high-level processing in continuous flash suppression. Trends Cogn. Sci. 23, 267-269 (2019).

53. Sterzer, P., Jalkanen, L. \& Rees, G. Electromagnetic responses to invisible face stimuli during binocular suppression. Neuroimage 46, 803-808 (2009).

54. Suzuki, M. \& Noguchi, Y. Reversal of the face-inversion effect in N170 under unconscious visual processing. Neuropsychologia 51, 400-409 (2013).

55. Schlossmacher, I., Junghöfer, M., Straube, T. \& Bruchmann, M. No differential effects to facial expressions under continuous flash suppression: An event-related potentials study. Neuroimage 163, 276-285 (2017).

56. Moradi, F., Koch, C. \& Shimojo, S. Face adaptation depends on seeing the face. Neuron 45, 169- 
175 (2005).

57. Amihai, I., Deouell, L. \& Bentin, S. Conscious awareness is necessary for processing race and gender information from faces. Conscious. Cogn. 20, 269-279 (2011).

58. Stein, T. \& Sterzer, P. High-level face shape adaptation depends on visual awareness: Evidence from continuous flash suppression. J. Vis. 11, (2011).

59. Nieuwenhuis, S. \& de Kleijn, R. Consciousness of targets during the attentional blink: A gradual or all-or-none dimension? Attention, Perception, Psychophys. 73, 364-373 (2011).

60. Overgaard, M., Rote, J., Mouridsen, K. \& Ramsøy, T. Z. Is conscious perception gradual or dichotomous? A comparison of report methodologies during a visual task. Conscious. Cogn. 15, 700-708 (2006).

61. Gayet, S., van Maanen, L., Heilbron, M., Paffen, C. L. E. \& Van der Stigchel, S. Visual input that matches the content of visual working memory requires less (not faster) evidence sampling to reach conscious access. J. Vis. 16, 1-20 (2016).

62. Shapiro, K. L., Arnell, K. M. \& Raymond, J. E. The attentional blink. Trends Cogn. Sci. 1, 291296 (1997).

63. Dehaene, S., Changeux, J. P., Naccache, L., Sackur, J. \& Sergent, C. Conscious, preconscious, and subliminal processing: A testable taxonomy. Trends Cogn. Sci. 10, 204-211 (2006).

64. Breitmeyer, B. G. Psychophysical 'blinding' methods reveal a functional hierarchy of unconscious visual processing. Conscious. Cogn. 35, 234-250 (2015).

65. Stein, T., Peelen, M. V. \& Sterzer, P. Adults' awareness of faces follows newborns' looking preferences. PLoS One 6, e29361 (2011).

66. Schmidt, T. \& Vorberg, D. Criteria for unconscious cognition: Three types of dissociation. Percept. Psychophys. 68, 489-504 (2006).

67. Reingold, E. M. \& Merikle, P. M. Using direct and indirect measures to study perception without awareness. Percept. Psychophys. 44, 563-575 (1988).

68. Vorberg, D., Mattler, U., Heinecke, A., Schmidt, T. \& Schwarzbach, J. Different time courses for visual perception and action priming. Proc. Natl. Acad. Sci. U. S. A. 100, 6275-6280 (2003).

69. Schmidt, T. The finger in flight: Real-time motor control by visually masked color stimuli. Psychol. Sci. 13, 112-118 (2002).

70. Ludwig, K., Sterzer, P., Kathmann, N., Franz, V. H. \& Hesselmann, G. Learning to detect but not to grasp suppressed visual stimuli. Neuropsychologia 51, 2930-2938 (2013).

71. Mastropasqua, T., Tse, P. U. \& Turatto, M. Learning of monocular information facilitates breakthrough to awareness during interocular suppression. Attention, Perception, Psychophys. 77, 790-803 (2015).

72. Gayet, S. \& Stein, T. Between-subject variability in the breaking continuous flash suppression 
paradigm: Potential causes, consequences, and solutions. Front. Psychol. 8, 437 (2017).

73. Paffen, C. L. E., Gayet, S., Heilbron, M. \& Van der Stigchel, S. Attention-based perceptual learning does not affect access to awareness. J. Vis. 18, 1-16 (2018).

74. Ramsøy, T. Z. \& Overgaard, M. Introspection and subliminal perception. Phenomenol. Cogn. Sci. 3, 1-23 (2004).

75. Franz, V. H. \& von Luxburg, U. No evidence for unconscious lie detection: A significant difference does not imply accurate classification. Psychol. Sci. 26, 1646-1648 (2015).

76. Battistoni, E., Stein, T. \& Peelen, M. V. Preparatory attention in visual cortex. Ann. N. Y. Acad. Sci. 1396, 92-107 (2017).

77. Gayet, S. et al. No evidence for mnemonic modulation of interocularly suppressed visual input. Neuroimage 215, 116801 (2020).

78. Fahrenfort, J. J. et al. Neuronal integration in visual cortex elevates face category tuning to conscious face perception. Proc. Natl. Acad. Sci. U. S. A. 109, 21504-21509 (2012).

79. Lamme, V. A. F. \& Roelfsema, P. R. The distinct modes of vision offered by feedforward and recurrent processing. Trends Neurosci. 23, 571-579 (2000).

80. Fahrenfort, J. J., Scholte, H. S. \& Lamme, V. A. F. Masking disrupts reentrant processing in human visual cortex. J. Cogn. Neurosci. 19, 1488-1497 (2007).

81. Yuval-Greenberg, S. \& Heeger, D. J. Continuous flash suppression modulates cortical activity in early visual cortex. J. Neurosci. 33, 9635-9643 (2013).

82. Crouzet, S. M., Kirchner, H. \& Thorpe, S. J. Fast saccades toward faces: Face detection in just 100 ms. J. Vis. 10, 1-17 (2010).

83. Fahrenfort, J. J., Van Leeuwen, J., Olivers, C. N. L. \& Hogendoorn, H. Perceptual integration without conscious access. Proc. Natl. Acad. Sci. U. S. A. 114, 3744-3749 (2017).

84. Moors, P., Boelens, D., van Overwalle, J. \& Wagemans, J. Scene integration without awareness: No conclusive evidence for processing scene congruency during continuous flash suppression. Psychol. Sci. 27, 945-956 (2016).

85. Stein, T., Reeder, R. R. \& Peelen, M. V. Privileged access to awareness for faces and objects of expertise. J. Exp. Psychol. Hum. Percept. Perform. 42, 788-798 (2016).

86. Kaiser, D., Quek, G. L., Cichy, R. M. \& Peelen, M. V. Object vision in a structured world. Trends Cogn. Sci. 23, 672-685 (2019).

87. Lamme, V. A. F. Why visual attention and awareness are different. Trends Cogn. Sci. 7, 12-18 (2003).

88. de Lange, F. P., Heilbron, M. \& Kok, P. How do expectations shape perception? Trends Cogn. Sci. 22, 764-779 (2018).

89. Summerfield, C. \& Egner, T. Expectation (and attention) in visual cognition. Trends Cogn. Sci. 
13, 403-409 (2009).

90. Kouider, S., de Gardelle, V., Sackur, J. \& Dupoux, E. How rich is consciousness? The partial awareness hypothesis. Trends Cogn. Sci. 14, 301-307 (2010).

91. Cohen, M. A., Cavanagh, P., Chun, M. M. \& Nakayama, K. The attentional requirements of consciousness. Trends in Cognitive Sciences vol. 16 411-417 (2012).

92. Bahrami, B., Lavie, N. \& Rees, G. Attentional load modulates responses of human primary visual cortex to invisible stimuli. Curr. Biol. 17, 509-513 (2007).

93. Van Boxtel, J. J. A., Tsuchiya, N. \& Koch, C. Opposing effects of attention and consciousness on afterimages. Proc. Natl. Acad. Sci. U. S. A. 107, 8883-8888 (2010).

94. Bahrami, B., Carmel, D., Walsh, V., Rees, G. \& Lavie, N. Unconscious orientation processing depends on perceptual load. J. Vis. 8, 1-10 (2008).

95. Naccache, L., Blandin, E. \& Dehaene, S. Unconscious masked priming depends on temporal attention. Psychol. Sci. 13, 416-424 (2002).

96. Bahrami, B., Carmel, D., Walsh, V., Rees, G. \& Lavie, N. Spatial attention can modulate unconscious orientation processing. Perception 37, 1520-1528 (2008).

97. Wyart, V. \& Tallon-Baudry, C. Neural dissociation between visual awareness and spatial attention. J. Neurosci. 28, 2667-2679 (2008).

98. Kanai, R., Tsuchiya, N. \& Verstraten, F. A. J. The scope and limits of top-down attention in unconscious visual processing. Curr. Biol. 16, 2332-2336 (2006).

99. Watanabe, M. et al. Attention but not awareness modulates the BOLD signal in the human V1 during binocular suppression. Science 334, 829-831 (2011).

100. Chelazzi, L., Miller, E. K., Duncan, J. \& Desimone, R. A neural basis for visual search in inferior temporal cortex. Nature 363, 345-347 (1993).

101. Bansal, A. K. et al. Neural dynamics underlying target detection in the human brain. J. Neurosci. 34, 3042-3055 (2014).

102. Miller, E. K. \& Cohen, J. D. An integrative theory of prefrontal cortex function. Annu. Rev. Neurosci. 24, 167-202 (2001).

103. Graziano, M. S. A. \& Webb, T. W. The attention schema theory: A mechanistic account of subjective awareness. Front. Psychol. 6, 500 (2015).

104. Peters, M. A. K. \& Lau, H. Human observers have optimal introspective access to perceptual processes even for visually masked stimuli. Elife 4, e09651 (2015).

105. Vadillo, M. A., Linssen, D., Orgaz, C., Parsons, S. \& Shanks, D. R. Unconscious or underpowered? Probabilistic cuing of visual attention. J. Exp. Psychol. Gen. 149, 160-181 (2020).

106. Vadillo, M. A., Konstantinidis, E. \& Shanks, D. R. Underpowered samples, false negatives, and unconscious learning. Psychon. Bull. Rev. 23, 87-102 (2016). 
107. Shanks, D. R. Regressive research: The pitfalls of post hoc data selection in the study of unconscious mental processes. Psychon. Bull. Rev. 24, 752-775 (2017).

108. Dienes, Z. How Bayesian statistics are needed to determine whether mental states are unconscious. in Behavioral Methods in Consciousness Research (ed. Overgaard, M.) 199-220 (Oxford Academic, 2015).

109. Brainard, D. H. The psychophysics toolbox. Spat. Vis. 10, 433-436 (1997).

110. Kelley, K. MBESS (Version 4.0.0 and higher). (2017).

111. Kelley, K. Confidence intervals for standardized effect sizes: Theory, application, and implementation. J. Stat. Softw. 20, 1-24 (2007).

112. Miles, W. R. Ocular dominance in human adults. J. Gen. Psychol. 3, 412-430 (1930).

113. Rabovsky, M., Stein, T. \& Abdel Rahman, R. Access to awareness for faces during continuous flash suppression is not modulated by affective knowledge. PLoS One 11, (2016).

114. Stein, T., Siebold, A. \& Van Zoest, W. Testing the idea of privileged awareness of self-relevant information. J. Exp. Psychol. Hum. Percept. Perform. 42, (2016).

115. Yang, E., Blake, R. \& McDonald, J. E. A new interocular suppression technique for measuring sensory eye dominance. Investig. Ophthalmol. Vis. Sci. 51, 588-593 (2010).

116. Macmillan, N. A. \& Creelman, C. D. Detection theory: A user's guide. (Lawrence Erlbaum, 2005).

117. Team, J. JASP (Version 0.12.2). (2020). 


\section{Acknowledgments}

We thank S. Gayet and Y. Pinto for suggestions on earlier versions of this manuscript, and D. Awad, T. Ciorli, C. Laurent, M. Leitjens, C. Riddell, F. Roelofs, and M. Wiggers for help with data collection. This project has received funding from the European Research Council (ERC) under the European Union's Horizon 2020 research and innovation programme (grant agreement No 725970). The funders had no role in study design, data collection and analysis, decision to publish or preparation of the manuscript.

\section{Author contributions}

T.S. and M.P. designed the study, interpreted the data, and drafted the paper. T.S. analysed the data.

\section{Competing interests}

The authors declare no competing interests. 

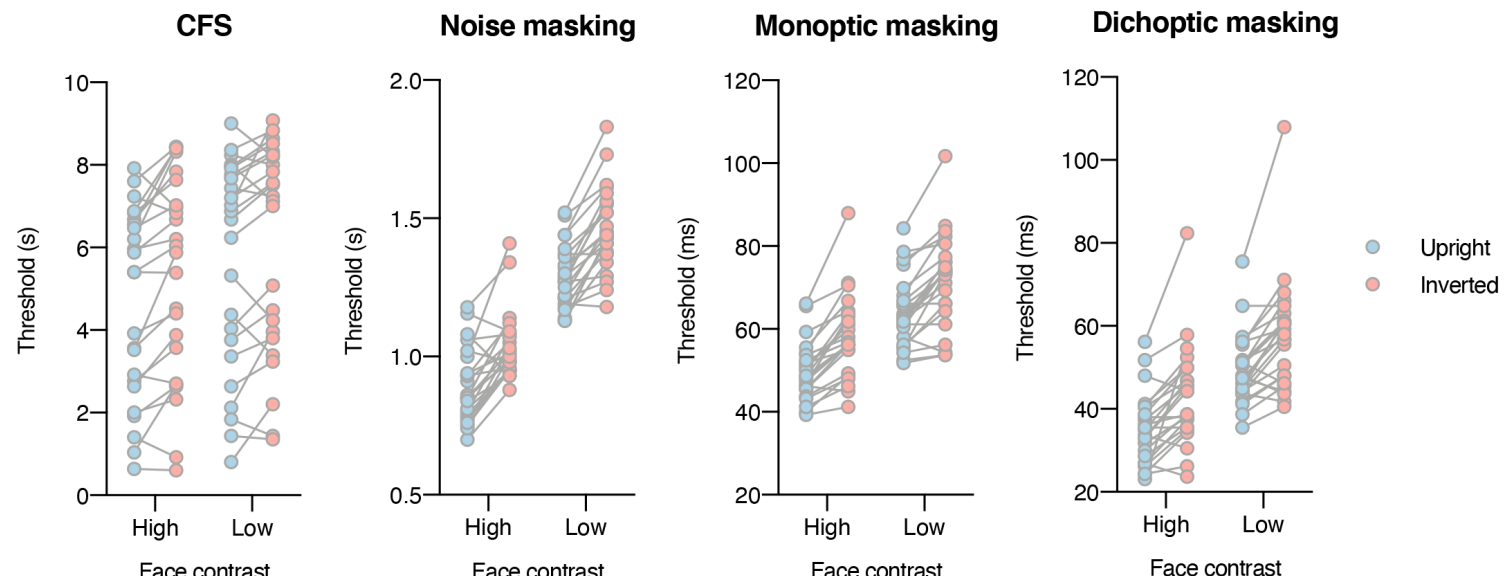

Extended Data Figure 1. Subject-level display of localization thresholds in Experiment 1. Localization thresholds for low- and high-contrast upright and inverted faces from the four detection paradigms. Note the different scales. Every circles represents an individual participant.
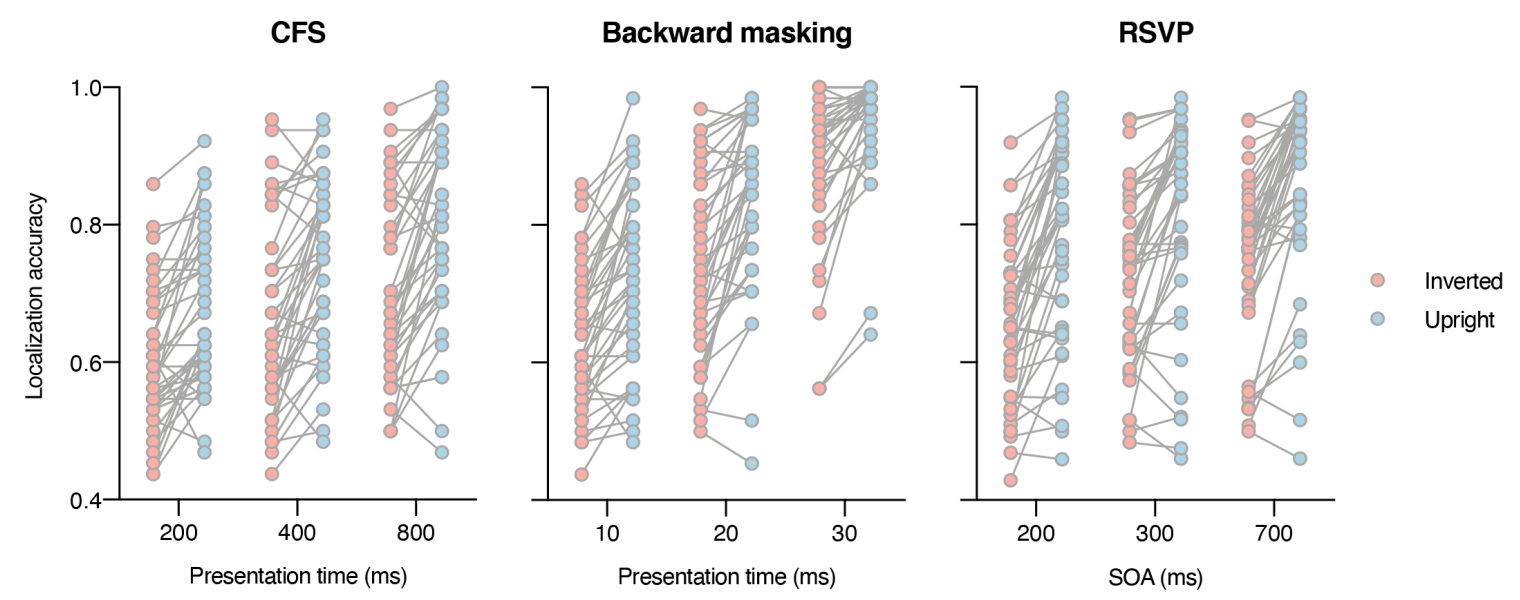

Extended Data Figure 2. Subject-level display of localization thresholds in Experiment 2. Localization accuracy for upright and inverted faces for the three different detection paradigms and presentation times. Every circles represents an individual participant. 


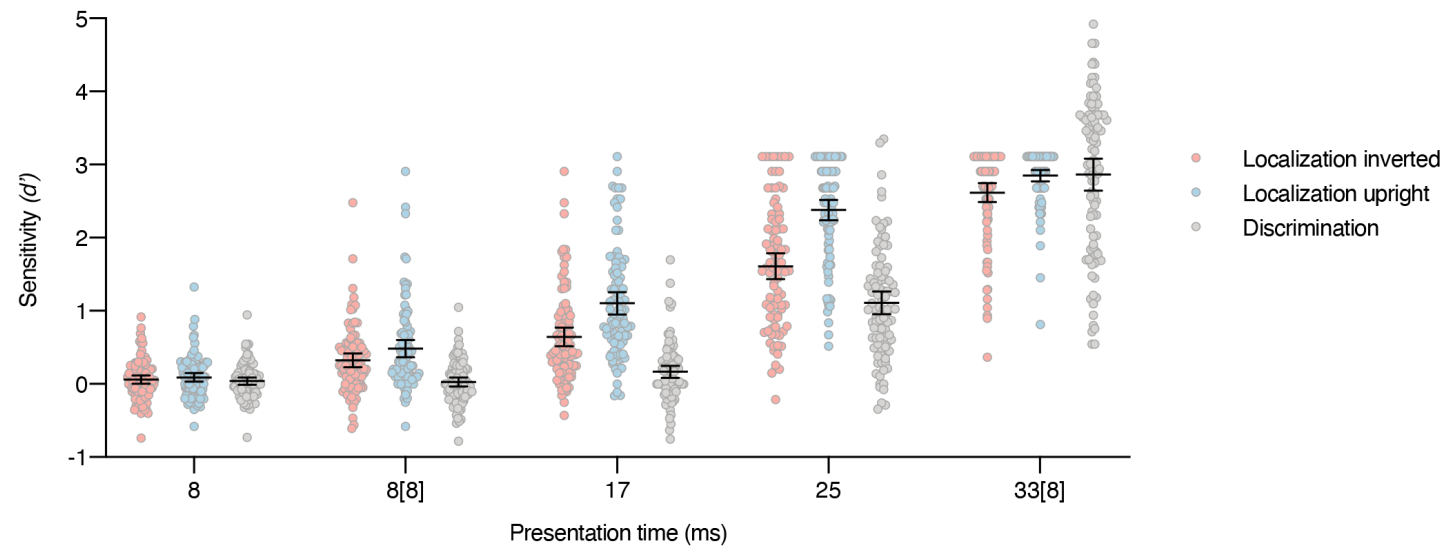

b

Detection vs. Discrimination

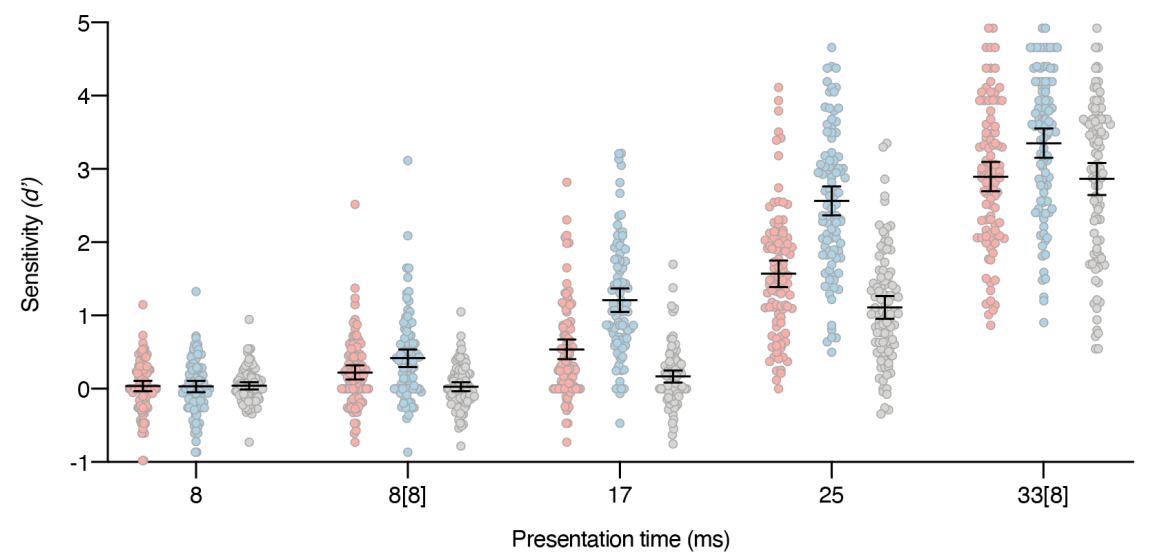

Detection inverted Detection upright

Discrimination

C

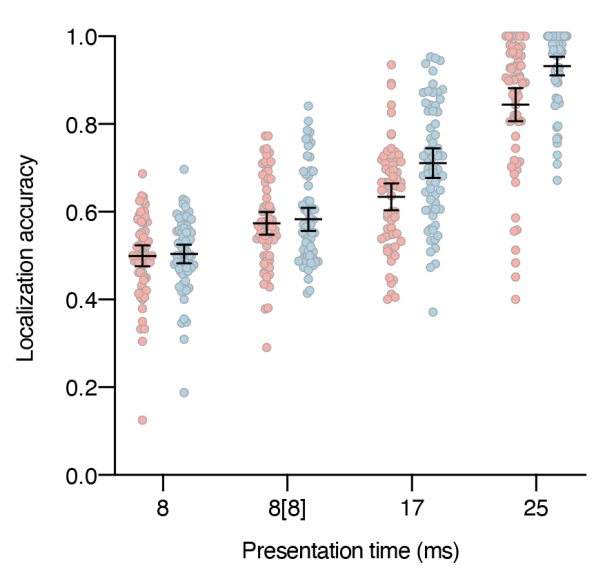

Discrimination incorrect

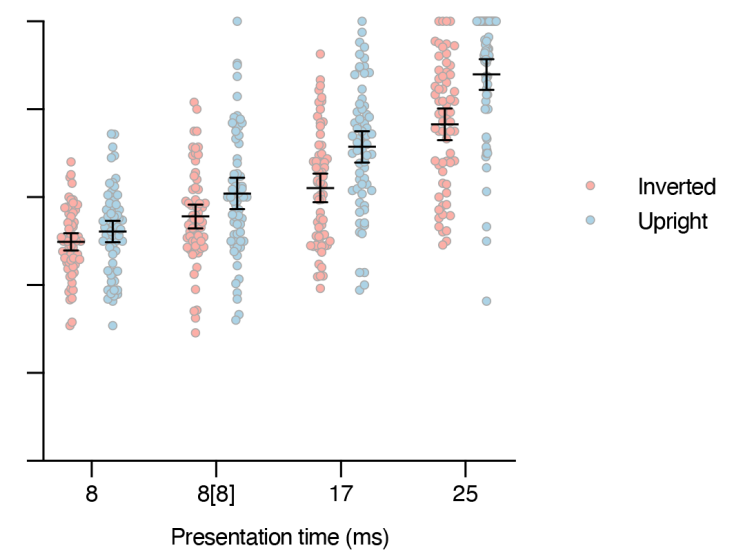

Extended Data Figure 3. Subject-level display of localization, detection and discrimination sensitivity

in Experiment 3. (a) Localization sensitivity and (b) detection sensitivity for upright and inverted faces for the five different presentation times (values in square brackets refer to an additional blank screen of 8 ms between the face stimulus and the mask). For comparison, both panels also show discrimination sensitivity. (c) Mean localization accuracy for upright and inverted faces shown for trials with correct (left panel) and incorrect (right panel) discrimination between upright and inverted faces. Every circle represents an individual participant, horizontal lines the means, and error bars $95 \% \mathrm{Cls}$. 


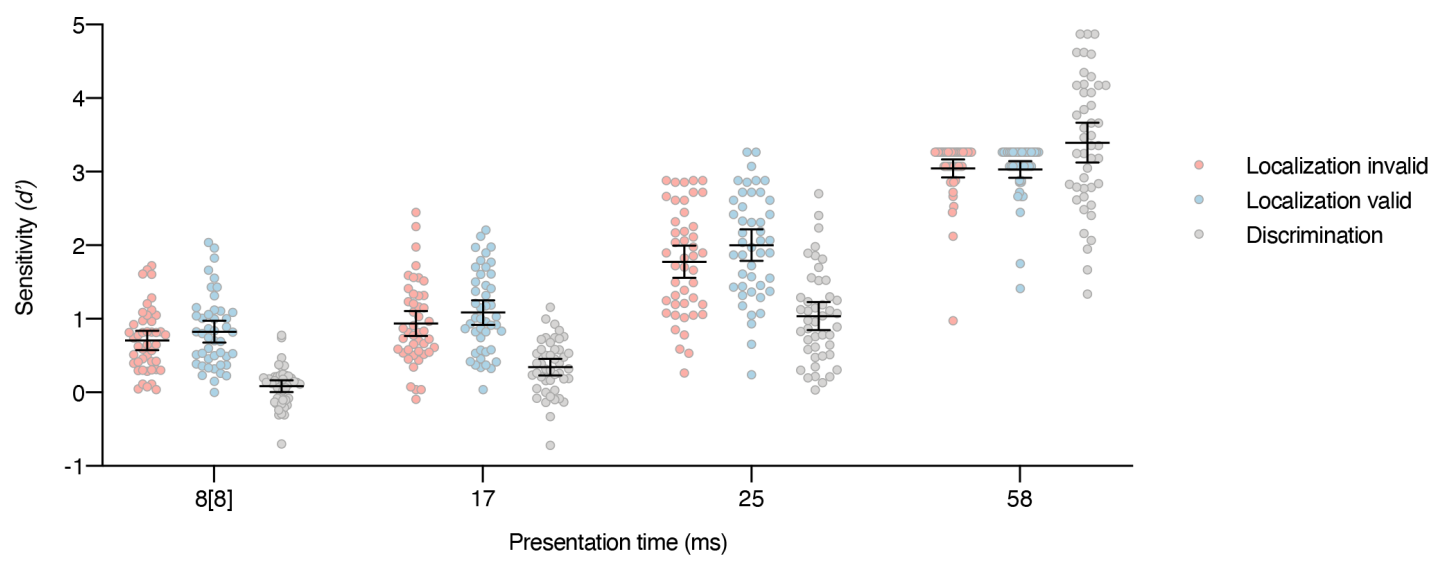

b

Experiment 4b: Localization vs. Discrimination

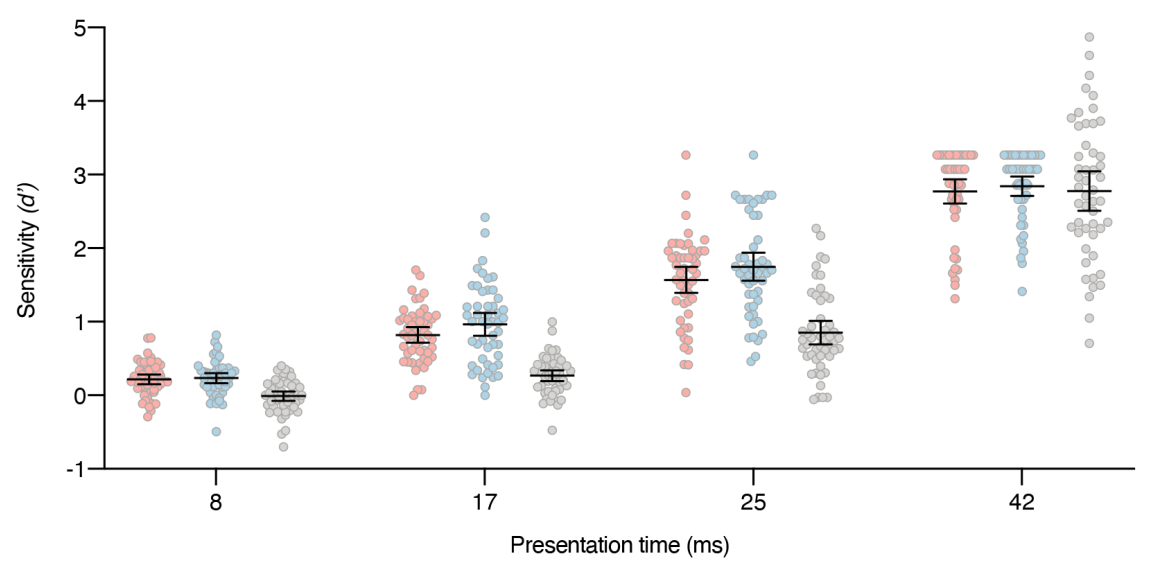

Localization invalid Localization valid Discrimination

c
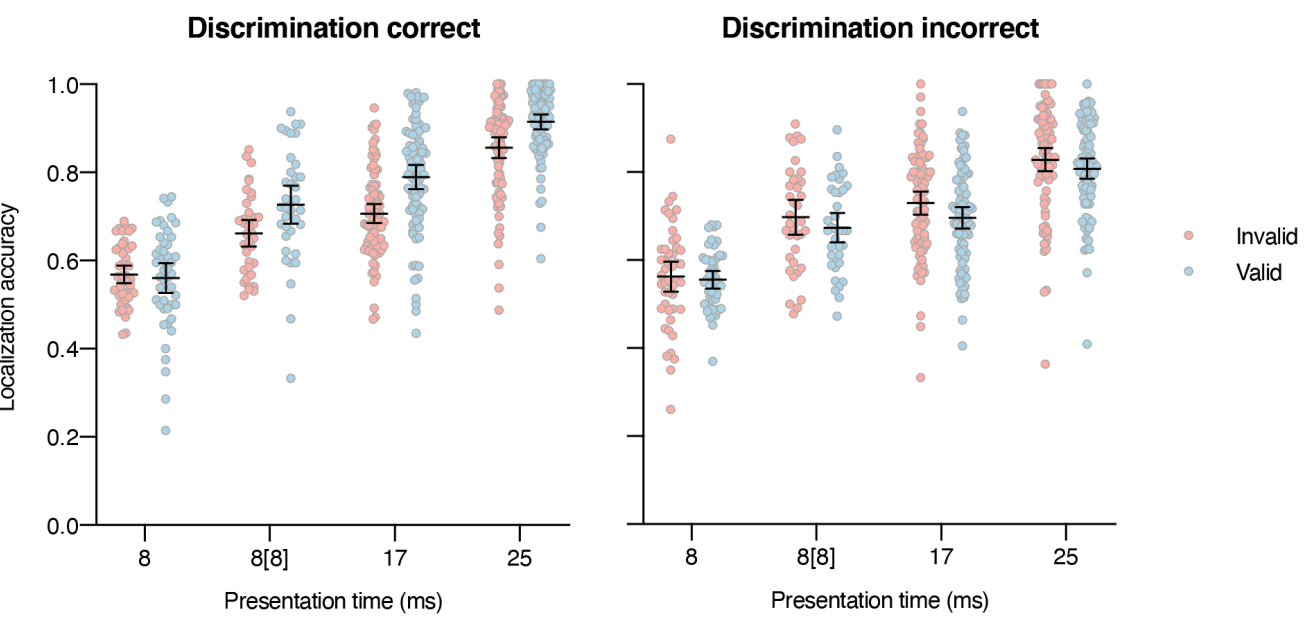

Extended Data Figure 4. Subject-level display of localization and discrimination sensitivity in Experiment 4. Localization and discrimination sensitivity for the four different presentation times in (a) Experiment $4 \mathrm{a}$ and (b) Experiment 4b. (c) Mean localization accuracy for validly and invalidly cued objects for trials with correct (left panel) and incorrect (right panel) discrimination between valid and invalid cues (collapsed across Experiment 4a and 4b). Every circle represents an individual participant, horizontal lines the means, and error bars $95 \% \mathrm{Cls}$. 


\section{Supplementary Discussion}

\section{Simultaneous noise masking vs. standard b-CFS control paradigm}

Instead of presenting faces transparently on top of CFS masks as is commonly done in standard b-CFS control paradigms (Supplementary Figure 1, top panel), in the simultaneous noise masking paradigm in Experiment 1 faces were faded-in on top of dynamically changing noise masks consisting of phasescrambled face stimuli (Supplementary Figure 1, bottom panel). This was done to prevent detection based on face-contours alone. Supplementary Figure 1 illustrates how faces can be detected based on their contour alone when faded in on top of CFS masks. By contrast, presentation against dynamic phase-scrambled noise yields a smooth subjective emergence of the whole face stimulus (contour and inner parts simultaneously). As upright and inverted faces have similar contours, we reasoned that detection based on the contour alone should mitigate a possible face-inversion effect, and this may be one reason why standard b-CFS control paradigms typically failed to yield effects. In actual CFS subjective face appearance is less predictable and faces are faded in against a grey background (the mask is presented to the other eye), such that contour-based detection may be a less viable strategy. Accordingly, we designed the masks adopted in the simultaneous noise masking paradigm in a way that rendered a detection strategy based on the contour alone less likely.

\section{Accuracy vs. response times for measuring detection effects}

Many previous b-CFS studies measured detection effects as differences in response times (RTs) using speeded tasks, for example by instructing participants to press a key as soon as possible to indicate on which side of fixation any part of the test stimulus became visible. Such speeded tasks require participants to set an internal criterion for responding, such that the resulting RTs are influenced by unknown (uncontrolled) factors such as response tendencies, confidence, uncertainty, etc. Thus, had we found detection effects similar to b-CFS in control paradigms with RTs, these effects could have reflected such uncontrolled factors (e.g. lower response criterion for upright than inverted faces). Instead of using speeded RT-based tasks, we thus recorded thresholds (Experiment 1) and accuracy/sensitivity (Experiments 2-4) with non-speeded tasks. Accuracy-based, forced-choice tasks such as the 2- and 4-AFC localization tasks employed in the present experiments are criterion-free, meaning that the results reflect differences in perceptual sensitivity (e.g. a lower response criterion for upright than inverted faces would not affect thresholds or accuracy/sensitivity). 


\section{Supplementary Figures}

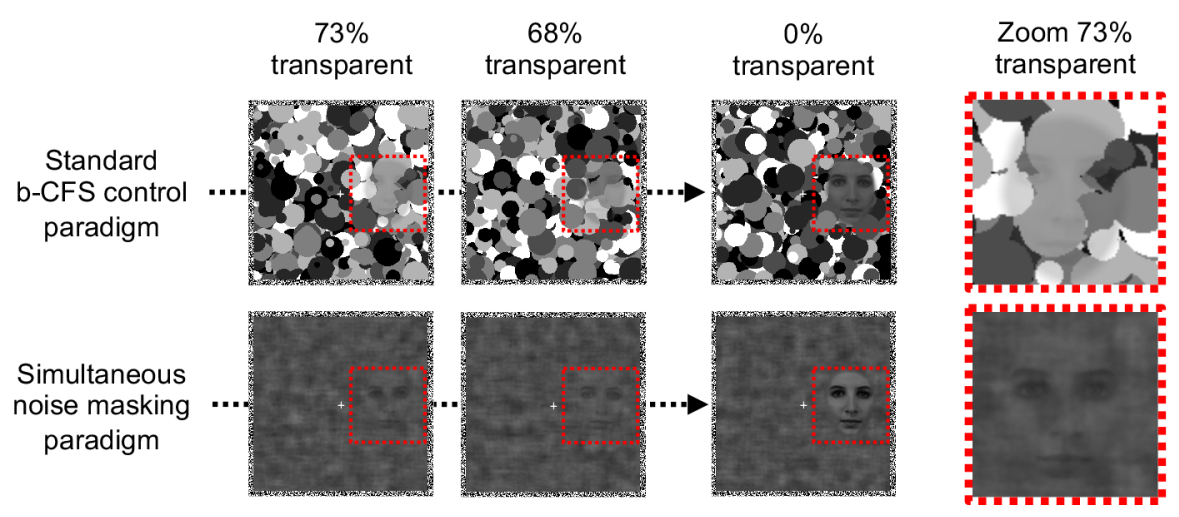

Supplementary Figure 1. Illustration of face-stimulus appearance. Top panel: In the standard b-CFS control paradigm the face stimulus is faded-in on top of CFS masks. Bottom panel: In the simultaneous noise masking paradigm from Experiment 1 the face stimulus is faded in on top of phase-scrambled noise masks. The different screenshots represent different levels of face transparency. In standard control paradigms, the face contour can often be detected before the inner facial features can be discerned (see the inset from a level of $73 \%$ transparency). In the simultaneous noise masking paradigm the face contour and inner facial features emerge from the noise around the same time. 


\section{Supplementary Results}

\section{Detailed statistical results}

For readability and brevity, in the main manuscript we did not report all statistical test results. Here, these additional details are provided, along with 95\% CIs for effect size estimates $d_{z}$ for $t$-tests.

\section{Experiment 1}

The effect of contrast was significant for CFS $\left(t(23)=5.48, p<.001, d_{z}=1.12,95 \%\right.$ CI for $d_{z}[0.60,1.62]$, $\left.\mathrm{BF}_{10}=1.57 \times 10^{3}\right)$, simultaneous noise masking $\left(t(23)=26.04, p<.001, d_{z}=5.32,95 \% \mathrm{CI}\right.$ for $d_{z}[3.73$, 6.89], $\left.\mathrm{BF}_{10}=2.70 \times 10^{15}\right)$, monoptic backward masking $\left(t(23)=17.55, p<.001, d_{z}=3.58,95 \%\right.$ CI for $d_{z}$ $\left.[2.47,4.68], \mathrm{BF}_{10}=7.16 \times 10^{11}\right)$, and dichoptic backward masking $\left(t(23)=20.60, p<.001, d_{z}=4.21,95 \%\right.$ CI for $\left.d_{z}[2.93,5.47], \mathrm{BF}_{10}=1.96 \times 10^{13}\right)$. Similarly, the effect of face inversion was significant for CFS $\left(t(23)=4.36, p<.001, d_{z}=0.89,95 \%\right.$ CI for $\left.d_{z}[0.41,1.36], \mathrm{BF}_{10}=128.13\right)$, simultaneous noise masking $\left(t(23)=10.82, p<.001, d_{z}=2.21,95 \% \mathrm{CI}\right.$ for $\left.d_{z}[1.45,2.95], \mathrm{BF}_{10}=5.89 \times 10^{7}\right)$, monoptic backward masking $\left(t(23)=7.97, p<.001, d_{z}=1.63,95 \% \mathrm{CI}\right.$ for $\left.d_{z}[1.01,2.24], \mathrm{BF}_{10}=3.13 \times 10^{5}\right)$, and dichoptic backward masking $\left(t(23)=5.63, p<.001, d_{z}=1.15,95 \% \mathrm{CI}\right.$ for $\left.d_{z}[0.62,1.66], \mathrm{BF}_{10}=2.17 \times 10^{3}\right)$.

\section{Experiment 2}

Averaged across all three presentation conditions, inversion effects were significant for CFS $(t(39)=7.70$, $p<.001, d_{z}=1.22,95 \% \mathrm{CI}$ for $\left.d_{z}[0.80,1.62], \mathrm{BF}_{10}=4.96 \times 10^{6}\right)$, backward masking $(t(39)=9.93, p<.001$, $d_{z}=1.57,95 \% \mathrm{CI}$ for $\left.d_{z}[1.10,2.03], \mathrm{BF}_{10}=2.81 \times 10^{9}\right)$, and RSVP $\left(t(39)=8.81, p<.001, d_{z}=1.39,95 \%\right.$ CI for $\left.d_{z}[0.95,1.83], \mathrm{BF}_{10}=1.24 \times 10^{8}\right)$. Inversion effects did not differ significantly between paradigms (mixed ANOVA, two-way interaction, $F(2,117)=1.82, p=.17, \eta_{p}^{2}=.03, \mathrm{BF}_{01}=3.25$ ) or presentation conditions $\left(\mathrm{CFS}: F(2,78)=1.18, p=.31, \eta_{p}^{2}=.03, \mathrm{BF}_{01}=7.05\right.$; backward masking: $F(2,78)=1.64, p=$ $.20, \eta_{p}^{2}=.04, \mathrm{BF}_{01}=4.39 ;$ RSVP: $\left.F(2,78)=1.75, p=.18, \eta_{p}^{2}=.04, \mathrm{BF}_{01}=7.22\right)$.

\section{Experiment 3}

Averaged across all presentation times, inversion effects were significant for both localization $(t(93)=$ 18.27, $p<.001, d_{z}=1.88,95 \% \mathrm{CI}$ for $\left.d_{z}[1.55,2.22], \mathrm{BF}_{10}=7.49 \times 10^{30}\right)$ and detection $(t(93)=19.23, p<$ $.001, d_{z}=1.98,95 \% \mathrm{CI}$ for $\left.d_{z}[1.63,2.33], \mathrm{BF}_{10}=1.84 \times 10^{29}\right)$. The interaction between presentation time and face orientation, reflecting larger inversion effects at intermediate presentation times, was significant for both localization $\left(F(4,372)=37.79, p<.001, \eta_{p}{ }^{2}=.29 ; \mathrm{BF}_{10}=9.72 \times 10^{15}\right)$ and detection $(F(4,372)=$ 80.88, $\left.p<.001, \eta_{p}^{2}=.47 ; \mathrm{BF}_{10}=4.11 \times 10^{17}\right)$. At the second shortest presentation time, orientation discrimination did not differ significantly from chance $\left(t(93)=0.91, p=.18\right.$ (one-tailed), $d_{z}=0.09,95 \%$ 
CI for $\left.d_{z}[-0.08, \infty], \mathrm{BF}_{0+}=3.59\right)$, while localization was significantly above chance both for upright faces $\left(t(93)=8.08, p<.001, d_{z}=0.83,95 \% \mathrm{CI}\right.$ for $\left.d_{z}[0.60,1.07], \mathrm{BF}_{10}=3.47 \times 10^{9}\right)$ and for inverted faces $(t(93)$ $=6.91, p<.001, d_{z}=0.71,95 \% \mathrm{CI}$ for $\left.d_{z}[0.49,0.94], \mathrm{BF}_{10}=1.70 \times 10^{7}\right)$. Also detection was significantly above chance at the second shortest presentation time, both for upright faces $\left(t(93)=6.93, p<.001, d_{z}\right.$ $=0.72,95 \% \mathrm{CI}$ for $\left.d_{z}[0.49,0.94], \mathrm{BF}_{10}=1.83 \times 10^{7}\right)$ and for inverted faces $\left(t(93)=4.64, p<.001, d_{z}=0.48\right.$, $95 \% \mathrm{CI}$ for $\left.d_{z}[0.26,0.69], \mathrm{BF}_{10}=1.41 \times 10^{3}\right)$. Localization sensitivity was significantly higher for upright than for inverted faces $\left(t(93)=4.11, p<.001, d_{z}=0.42,95 \% \mathrm{CI}\right.$ for $\left.d_{z}[0.21,0.63], \mathrm{BF}_{10}=220.59\right)$. Also detection sensitivity was significantly higher for upright than for inverted faces $(t(93)=4.69, p<.001$, $d_{z}=0.48,95 \% \mathrm{CI}$ for $\left.d_{z}[0.27,0.70], \mathrm{BF}_{10}=1.73 \times 10^{3}\right)$. Inversion effects exceeded orientation discrimination, both for localization $\left(t(93)=2.82, p=.006, d_{z}=0.29,95 \% \mathrm{CI}\right.$ for $d_{z}[0.08,0.50], \mathrm{BF}_{10}=$ $4.67)$ and for detection $\left(t(93)=3.21, p=.002, d_{z}=0.33,95 \% \mathrm{CI}\right.$ for $\left.d_{z}[0.12,0.54], \mathrm{BF}_{10}=13.16\right)$. In those 64 participants who had enough trials with correct and incorrect discrimination responses for every condition, localization accuracy for upright faces was significantly better than for inverted faces for both trials with correct discrimination responses $\left(F(1,63)=54.38, p<.001, \eta_{p}{ }^{2}=.46, \mathrm{BF}_{10}=1.17 \times 10^{5}\right)$ and trials with incorrect discrimination responses $\left(F(1,63)=58.24, p<.001, \eta_{p}^{2}=.48, \mathrm{BF}_{10}=6.72 \times 10^{10}\right)$. Similarly, detection accuracy for upright faces was significantly better than for inverted faces for both trials with correct discrimination responses $\left(F(1,63)=102.61, p<.001, \eta_{p}^{2}=.62, \mathrm{BF}_{10}=1.24 \times 10^{12}\right)$ and trials with incorrect discrimination responses $\left(F(1,63)=88.82, p<.001, \eta_{p}^{2}=.59, \mathrm{BF}_{10}=3.35 \times 10^{20}\right)$.

\section{Experiment 4}

In Experiment 4a, at the shortest presentation time ( $8 \mathrm{~ms}$, followed by a blank of $8 \mathrm{~ms}$ ) the cueing effect was significant $\left(t(44)=2.95, p=.005, d_{z}=0.44,95 \% \mathrm{CI}\right.$ for $\left.d_{z}[0.13,0.74], \mathrm{BF}_{10}=6.97\right)$; however, also cue discriminability exceeded chance performance $\left(t(44)=2.08, p=.022\right.$, one-tailed, $d_{z}=0.31,95 \% \mathrm{CI}$ for $d_{z}[0.06, \infty], \mathrm{BF}_{0+}=0.45$ ). In Experiment $4 \mathrm{~b}$, at the shortest presentation time ( $8 \mathrm{~ms}$, no blank) cue discriminability did not differ significantly from chance $\left(t(49)=-0.35, p=.36\right.$, one-tailed, $d_{z}=-0.05$, $95 \% \mathrm{CI}$ for $\left.d_{z}[-0.28, \infty], \mathrm{BF}_{0+}=8.36\right)$, while localization sensitivity was significantly above chance for validly cued objects $\left(t(49)=6.91, p<.001, d_{z}=0.98 ; 95 \% \mathrm{CI}\right.$ for $\left.d_{z}[0.64,1.31], \mathrm{BF}_{10}=1.35 \times 10^{6}\right)$ as well as for invalidly cued objects $\left(t(49)=6.68, p<.001, d_{z}=0.95 ; 95 \% \mathrm{CI}\right.$ for $\left.d_{z}[0.61,1.28], \mathrm{BF}_{10}=6.30 \times 10^{5}\right)$. However, there was no significant difference in localization sensitivity between validly and invalidly cued objects $\left(t(49)=0.36, p=.72, d_{z}=0.05,95 \% \mathrm{CI}\right.$ for $\left.d_{z}[-0.23,0.33], \mathrm{BF}_{01}=6.11\right)$.

\section{Analyses of correct vs. incorrect discrimination responses}

In the main manuscript, we report analyses of trials with correct and incorrect discrimination responses for Experiment 3 and 4 across all presentation times except the longest for those participants who had 
at least five trials per condition (stimulus $\times$ presentation time). To ensure that these results did not depend on these specific inclusion criteria, here we report localization accuracy for different inclusion criteria. We report results when including participants who had at least four, six, or eight trials per condition, and we report separate analyses for only those presentation times where discrimination was not significantly different from chance (discrimination $\approx 0$ ) and for those presentation times where discrimination was significantly above chance (discrimination $>0$ ). For Experiment 3 , these additional analyses are reported in Supplementary Table 1; for Experiment 4 in Supplementary Table 2.

\section{Experiment 3}

As can be seen in Supplementary Table 1, for the analyses across all presentation times except the longest (as reported in the main manuscript) for all inclusion criteria inversion effects were significant for trials with both correct and incorrect discrimination responses. The specific participant inclusion criterion had little effect. For some inclusion criteria a significant interaction between correctness of the discrimination response and face orientation reflected somewhat larger effects for trials with incorrect discrimination responses. For the two longer presentation times (17 ms and $25 \mathrm{~ms}$ ), where discrimination was significantly above chance (discrimination $>0$ ), inversion effects were similarly strong for trials with correct and incorrect discrimination responses. For the two shortest presentation times ( $8 \mathrm{~ms}$ and $8[8] \mathrm{ms}$ ), where discrimination was not significantly above chance (discrimination $\approx$ 0 ), somewhat surprisingly, inversion effects were restricted to trials with incorrect discrimination responses and were not significant for trials with correct discrimination responses. However, this observation should be interpreted with caution, as the interactions between correctness of the discrimination response and face orientation were not always significant and Bayes factors provided only anecdotal evidence for the interaction effect. In summary, these results further support the conclusion that the face-inversion effect does not depend on awareness (discriminability) of face orientation.

\section{Experiment 4}

To increase power we collapsed the data from the identical presentation conditions of 17 and $25 \mathrm{~ms}$ (discrimination $>0$ ) from Experiment $4 \mathrm{a}$ and $4 \mathrm{~b}$ (thus dropping the 8[8]-ms condition from Experiment 4a, see the end of this section for this condition). For all inclusion criteria, there was strong evidence for a cueing effect in trials with correct discrimination responses. A significant interaction between correctness of the discrimination response and cue validity indicated that this cueing effect was absent in trials with incorrect discrimination responses: Here, the effect even tended to reverse, with significantly better localization accuracy in invalid than in valid trials. For the presentation time $(8 \mathrm{~ms})$ from Experiment $4 \mathrm{~b}$ where cue discriminability was not significantly above chance (discrimination $\approx$ 
0 ), there was no evidence for a cueing effect. These results further establish that some awareness of cue validity is required for the effect of category-based attention.

Finally, to directly test the effect of cue discriminability across experiments, we conducted an additional comparison of cueing effects for the shortest presentation times in Experiment 4a (8[8] ms, where discrimination was significantly above chance), and in Experiment $4 \mathrm{~b}(8 \mathrm{~ms}$, where discrimination was not significantly different from chance) as a function of the correctness of the discrimination response. Here, a significant three-way interaction in a mixed ANOVA with the between-subject factor experiment and the within-subject factors correctness of the discrimination response and cue validity indicates that cue discriminability influenced the cueing effect differently between experiments, i.e. that only in Experiment 4a, where overall cue discriminability was above chance (and thus discrimination responses were informative), cueing effects were modulated by conscious access to cue validity. With the inclusion criterion of at least five trials per condition (Experiment 4a: 42 participants, Experiment $4 \mathrm{~b}: 48$ participants) this three-way interaction was significant $\left(F(1,88)=8.24, p=.005, \eta_{p}^{2}=.09, \mathrm{BF}_{10}=\right.$ 4.07). The four-trials inclusion criteria resulted in the same group of participants; for the six-trials inclusion criterion (Experiment 4a: 42 participants, Experiment 4b: 47 participants) and for the eighttrials inclusion criterion (Experiment 4a: 39 participants, Experiment $4 \mathrm{~b}: 46$ participants) the results were nearly identical $\left(F(1,87)=8.22, p=.005, \eta_{p}{ }^{2}=.09, \mathrm{BF}_{10}=4.50\right.$, and $F(1,83)=9.63, p=.003, \eta_{p}{ }^{2}=$ $.10, \mathrm{BF}_{10}=8.58$, respectively). To unpack this three-way interaction: At the shortest presentation time in Experiment $4 \mathrm{~b}(8 \mathrm{~ms})$, there was no evidence that cueing effects were modulated by the correctness of the discrimination response (see Table S2), while at the shortest presentation time in Experiment $4 \mathrm{a}$ $(8[8] \mathrm{ms})$, this interaction was significant, for the identical four-, five-, and six-trials inclusion criteria $\left(F(1,41)=12.07, p=.001, \eta_{p}{ }^{2}=.23, \mathrm{BF}_{10}=91.64\right)$, and for the eight-trials inclusion criterion $(F(1,38)=$ 14.27, $\left.p<.001, \eta_{p}^{2}=.27, \mathrm{BF}_{10}=237.61\right)$ : Cueing effects were larger in trials with correct than with incorrect discrimination responses. 
Supplementary Table 1. Additional results from Experiment 3

\begin{tabular}{|c|c|c|c|c|}
\hline & $\begin{array}{l}\text { Minimum no. of } \\
\text { trials } \\
\text { (No. of participants) }\end{array}$ & $\begin{array}{c}\text { Correct } \\
\text { discrimination }\end{array}$ & $\begin{array}{c}\text { Incorrect } \\
\text { discrimination }\end{array}$ & $\begin{array}{l}\text { Interaction } \\
\text { (correctness } \times \\
\text { orientation) }\end{array}$ \\
\hline \multirow{4}{*}{$\begin{array}{l}\text { All presentation } \\
\text { times (excluding } \\
\text { the longest) }\end{array}$} & $4(66)$ & $\begin{array}{c}\text { Upr } 68.3 \%, \text { inv } 64.0 \% \\
F(1,65)=51.19, p<.001 \\
\eta_{p}^{2}=.44, \mathrm{BF}_{10}=1.00 \times 10^{5}\end{array}$ & $\begin{array}{c}\text { Upr } 68.2 \%, \text { inv } 61.1 \% \\
F(1,65)=63.35, p<.001 \\
\eta_{p}^{2}=.49, B_{10}=3.44 \times 10^{11}\end{array}$ & $\begin{array}{c}F(1,65)=5.54, p=.022 \\
\eta_{p}^{2}=.08, B_{10}=1.67\end{array}$ \\
\hline & $5(64)$ & $\begin{array}{c}\text { Upr } 68.2 \%, \text { inv } 63.8 \% \\
F(1,63)=54.38, p<.001 \\
\eta_{p}^{2}=.46, \mathrm{BF}_{10}=1.17 \times 10^{5}\end{array}$ & $\begin{array}{c}\text { Upr } 68.1 \%, \text { inv } 61.0 \% \\
F(1,63)=58.24, p<.001 \\
\eta_{p}^{2}=.48, B_{10}=6.72 \times 10^{10}\end{array}$ & $\begin{array}{c}F(1,63)=4.57, p=.036 \\
\eta_{p}^{2}=.07, B_{10}=0.94\end{array}$ \\
\hline & $6(62)$ & $\begin{array}{c}\text { Upr } 68.1 \%, \text { inv } 63.7 \% \\
F(1,61)=53.67, p<.001 \\
\eta_{p}^{2}=.47, \mathrm{BF}_{10}=7.90 \times 10^{4}\end{array}$ & $\begin{array}{c}\text { Upr } 67.8 \%, \text { inv } 60.9 \% \\
F(1,61)=53.60, p<.001 \\
\eta_{p}^{2}=.47, \mathrm{BF}_{10}=1.52 \times 10^{10}\end{array}$ & $\begin{array}{l}F(1,61)=20.90, p<.001 \\
\eta_{p}^{2}=.26, B_{10}=1.04 \times 10^{4}\end{array}$ \\
\hline & $8(55)$ & $\begin{array}{c}\text { Upr } 67.6 \%, \text { inv } 63.3 \% \\
F(1,54)=41.03, p<.001 \\
\eta_{p}^{2}=.43, \mathrm{BF}_{10}=8.37 \times 10^{3}\end{array}$ & $\begin{array}{c}\text { Upr } 67.5 \%, \text { inv } 60.7 \% \\
F(1,54)=48.63, p<.001 \\
\eta_{p}^{2}=.47, B_{10}=4.42 \times 10^{8}\end{array}$ & $\begin{array}{c}F(1,54)=3.63, p=.062 \\
\eta_{p}^{2}=.06, B_{10}=0.73\end{array}$ \\
\hline \multirow{4}{*}{ Discrimination $>0$} & $4(80)$ & $\begin{array}{c}\text { Upr } 84.0 \%, \text { inv } 76.5 \% \\
F(1,79)=62.19, p<.001 \\
\eta_{p}^{2}=.44, B_{10}=5.86 \times 10^{9}\end{array}$ & $\begin{array}{c}\text { Upr } 81.5 \%, \text { inv } 71.0 \% \\
F(1,79)=77.59, p<.001 \\
\eta_{p}^{2}=.50, B_{10}=4.68 \times 10^{14}\end{array}$ & $\begin{array}{c}F(1,79)=3.74, p=.057 \\
\eta_{p}{ }^{2}=.05, \mathrm{BF}_{10}=1.00\end{array}$ \\
\hline & $5(77)$ & $\begin{array}{c}\text { Upr } 83.7 \%, \text { inv } 76.3 \% \\
F(1,76)=58.52, p<.001 \\
\eta_{p}^{2}=.44, B_{10}=1.29 \times 10^{9}\end{array}$ & $\begin{array}{c}\text { Upr } 81.5 \%, \text { inv } 71.0 \% \\
F(1,76)=72.27, p<.001 \\
\eta_{p}^{2}=.49, B_{10}=1.16 \times 10^{14}\end{array}$ & $\begin{array}{c}F(1,76)=3.52, p=.065 \\
\eta_{p}{ }^{2}=.04, B_{10}=1.22\end{array}$ \\
\hline & $6(72)$ & $\begin{array}{c}\text { Upr } 83.2 \%, \text { inv } 75.2 \% \\
F(1,71)=65.33, p<.001 \\
\eta_{p}^{2}=.48, B_{10}=1.27 \times 10^{10}\end{array}$ & $\begin{array}{c}\text { Upr } 80.5 \%, \text { inv } 70.2 \% \\
F(1,71)=62.16, p<.001 \\
\eta_{p}^{2}=.47, \mathrm{BF}_{10}=1.30 \times 10^{12}\end{array}$ & $\begin{array}{c}F(1,71)=1.79, p=.185 \\
\eta_{p}^{2}=.03, B_{10}=0.39\end{array}$ \\
\hline & $8(62)$ & $\begin{array}{c}\text { Upr } 82.5 \%, \text { inv } 74.5 \% \\
F(1,61)=54.24, p<.001 \\
\eta_{p}^{2}=.47, \mathrm{BF}_{10}=1.41 \times 10^{8}\end{array}$ & $\begin{array}{c}\text { Upr } 80.4 \%, \text { inv } 69.9 \% \\
F(1,61)=53.52, p<.001 \\
\eta_{p}^{2}=.47, B_{10}=3.02 \times 10^{10}\end{array}$ & $\begin{array}{c}F(1,61)=1.78, p=.188 \\
\eta_{p}^{2}=.03, B_{10}=0.43\end{array}$ \\
\hline \multirow{4}{*}{ Discrimination $\approx 0$} & $4(84)$ & $\begin{array}{c}\text { Upr } 56.7 \%, \text { inv } 56.1 \% \\
F(1,83)=0.42, p=.520 \\
\eta_{p}{ }^{2}<.01, B_{10}=0.16\end{array}$ & $\begin{array}{c}\text { Upr } 58.3 \%, \text { inv } 54.1 \% \\
F(1,83)=15.53, p<.001 \\
\eta_{p}^{2}=.16, B_{10}=73.28\end{array}$ & $\begin{array}{c}F(1,83)=5.91, p=.017 \\
\eta_{p}^{2}=.07, \mathrm{BF}_{10}=1.39\end{array}$ \\
\hline & $5(80)$ & $\begin{array}{c}\text { Upr } 56.8 \%, \text { inv } 56.2 \% \\
F(1,79)=0.36, p=.552 \\
\eta_{p}{ }^{2}<.01, B_{10}=0.15\end{array}$ & $\begin{array}{c}\text { Upr } 58.3 \%, \text { inv } 54.2 \% \\
F(1,79)=13.63, p<.001 \\
\eta_{p}^{2}=.15, B_{10}=50.87\end{array}$ & $\begin{array}{c}F(1,79)=4.95, p=.029 \\
\eta_{p}^{2}=.06, B_{10}=1.45\end{array}$ \\
\hline & $6(79)$ & $\begin{array}{c}\text { Upr } 56.6 \%, \text { inv } 55.7 \% \\
F(1,78)=0.93, p=.337 \\
\eta_{p}{ }^{2}=.01, B_{10}=0.20\end{array}$ & $\begin{array}{c}\text { Upr } 57.9 \%, \operatorname{inv} 54.1 \% \\
F(1,78)=12.38, p<.001 \\
\eta_{p}^{2}=.14, B_{10}=28.13\end{array}$ & $\begin{array}{c}F(1,78)=3.90, p=.052 \\
\eta_{p}^{2}=.05, B_{10}=0.72\end{array}$ \\
\hline & $8(75)$ & $\begin{array}{c}\text { Upr } 56.8 \%, \text { inv } 56.1 \% \\
F(1,74)=0.51, p=.476 \\
\eta_{p}{ }^{2}<.01, B_{10}=0.16\end{array}$ & $\begin{array}{c}\text { Upr } 57.9 \%, \text { inv } 54.2 \% \\
F(1,74)=10.85, p=.002 \\
\eta_{p}^{2}=.13, B_{10}=19.75\end{array}$ & $\begin{array}{c}F(1,74)=3.94, p=.053 \\
\eta_{p}^{2}=.05, B_{10}=0.97\end{array}$ \\
\hline
\end{tabular}

Face-inversion effects for trials with correct and incorrect discrimination responses. Shown are marginal means (percentage correct localization accuracy) for upright and inverted faces, separately for trials with correct and incorrect discrimination responses, and the associated main effect of face orientation, as well as the interaction between correctness of the discrimination response and face orientation. Different rows show different participant inclusion criteria. 
Supplementary Table 2. Additional results from Experiment 4

\begin{tabular}{|c|c|c|c|c|}
\hline & $\begin{array}{l}\text { Minimum no. of } \\
\text { trials } \\
\text { (No. of participants) }\end{array}$ & $\begin{array}{c}\text { Correct } \\
\text { discrimination }\end{array}$ & $\begin{array}{c}\text { Incorrect } \\
\text { discrimination }\end{array}$ & $\begin{array}{l}\text { Interaction } \\
\text { (correctness } \times \text { cue } \\
\text { validity) }\end{array}$ \\
\hline \multirow[t]{4}{*}{$\begin{array}{c}\text { Discrimination }>0 \\
\text { (Experiment } 4 \mathrm{a} \text { and } \\
4 \mathrm{~b})\end{array}$} & $4(91)$ & $\begin{array}{l}\text { Valid } 85.9 \%, \text { invalid } 78.6 \% \\
F(1,90)=89.40, p<.001 \\
\eta_{p}^{2}=.50, B_{10}=4.69 \times 10^{20}\end{array}$ & $\begin{array}{l}\text { Valid } 75.9 \% \text {, invalid } 78.4 \% \\
\qquad \begin{array}{c}F(1,90)=8.92, p=.004 \\
\eta_{p}^{2}=.09, \mathrm{BF}_{10}=9.68\end{array}\end{array}$ & $\begin{array}{l}F(1,90)=61.84, p<.001 \\
\eta_{p}^{2}=.41, B_{10}=1.30 \times 10^{16}\end{array}$ \\
\hline & $5(90)$ & $\begin{array}{l}\text { Valid } 85.8 \%, \text { invalid } 78.4 \% \\
F(1,89)=87.82, p<.001 \\
\eta_{p}^{2}=.50, B_{10}=3.29 \times 10^{20}\end{array}$ & $\begin{array}{l}\text { Valid } 75.7 \% \text {, invalid } 78.2 \% \\
\qquad \begin{array}{c}F(1,89)=8.49, p=.005 \\
\eta_{p}^{2}=.09, \mathrm{BF}_{10}=7.41\end{array}\end{array}$ & $\begin{array}{l}F(1,89)=60.24, p<.001 \\
\eta_{p}^{2}=.40, B_{10}=7.81 \times 10^{15}\end{array}$ \\
\hline & $6(89)$ & $\begin{array}{l}\text { Valid } 85.7 \%, \text { invalid } 78.3 \% \\
F(1,88)=88.31, p<.001 \\
\eta_{p}^{2}=.50, B_{10}=3.77 \times 10^{20}\end{array}$ & $\begin{array}{l}\text { Valid } 75.5 \% \text {, invalid } 78.1 \% \\
\qquad \begin{array}{c}F(1,88)=9.06, p=.003 \\
\eta_{p}^{2}=.09, \mathrm{BF}_{10}=9.82\end{array}\end{array}$ & $\begin{array}{l}F(1,88)=61.99, p<.001 \\
\eta_{p}^{2}=.41, B_{10}=1.64 \times 10^{16}\end{array}$ \\
\hline & $8(84)$ & $\begin{array}{l}\text { Valid } 85.4 \%, \text { invalid } 78.4 \% \\
F(1,83)=76.32, p<.001 \\
\eta_{p}^{2}=.48, B_{10}=3.72 \times 10^{17}\end{array}$ & $\begin{array}{c}\text { Valid } 75.6 \% \text {, invalid } 77.8 \% \\
\qquad \begin{array}{c}F(1,83)=6.36, p=.014 \\
\eta_{p}^{2}=.07, \mathrm{BF}_{10}=2.67\end{array}\end{array}$ & $\begin{array}{l}F(1,83)=52.60, p<.001 \\
\eta_{p}^{2}=.39, \mathrm{BF}_{10}=7.02 \times 10^{12}\end{array}$ \\
\hline \multirow[t]{4}{*}{$\begin{array}{l}\text { Discrimination } \approx 0 \\
\text { (Experiment } 4 b)\end{array}$} & $4(48)$ & $\begin{array}{c}\text { Valid } 56.1 \% \text {, invalid } 56.8 \% \\
F(1,47)=0.17, p=.686 \\
\eta_{p}^{2}<.01, B_{10}=0.23\end{array}$ & $\begin{array}{c}\text { Valid } 55.8 \% \text {, invalid } 56.4 \% \\
F(1,47)=0.14, p=.715 \\
\eta_{p}^{2}<.01, \mathrm{BF}_{10}=0.23\end{array}$ & $\begin{array}{c}F(1,47)<0.01, p=.956 \\
\eta_{p}^{2}<.01, \mathrm{BF}_{10}=0.21\end{array}$ \\
\hline & $5(48)$ & $\begin{array}{c}\text { Valid } 56.1 \% \text {, invalid } 56.8 \% \\
\qquad \begin{array}{c}F(1,47)=0.17, p=.686 \\
\eta_{p}^{2}<.01, B_{10}=0.23\end{array}\end{array}$ & $\begin{array}{c}\text { Valid } 55.8 \% \text {, invalid } 56.4 \% \\
F(1,47)=0.14, p=.715 \\
\eta_{p}^{2}<.01, \mathrm{BF}_{10}=0.23\end{array}$ & $\begin{array}{c}F(1,47)<0.01, p=.956 \\
\eta_{p}^{2}<.01, B_{10}=0.21\end{array}$ \\
\hline & $6(47)$ & $\begin{array}{c}\text { Valid } 56.4 \%, \text { invalid } 56.8 \% \\
\qquad \begin{array}{c}F(1,46)=0.06, p=.814 \\
\eta_{p}^{2}<.01, B_{10}=0.22\end{array}\end{array}$ & $\begin{array}{c}\text { Valid } 55.9 \% \text {, invalid } 56.2 \% \\
\qquad \begin{array}{c}F(1,46)=0.02, p=.893 \\
\eta_{p}^{2}<.01, \mathrm{BF}_{10}=0.22\end{array}\end{array}$ & $\begin{array}{c}F(1,46)<0.01, p=.923 \\
\eta_{p}^{2}<.01, \mathrm{BF}_{10}=0.21\end{array}$ \\
\hline & $8(46)$ & $\begin{array}{c}\text { Valid } 57.0 \% \text {, invalid } 57.0 \% \\
F(1,45)<0.01, p=.993 \\
\eta_{p}^{2}<.01, B_{10}=0.22\end{array}$ & $\begin{array}{c}\text { Valid } 56.0 \% \text {, invalid } 55.8 \% \\
F(1,45)=0.01, p=.910 \\
\eta_{p}{ }^{2}<.01, \mathrm{BF}_{10}=0.21\end{array}$ & $\begin{array}{c}F(1,45)<0.01, p=.923 \\
\eta_{p}^{2}<.01, \mathrm{BF}_{10}=0.22\end{array}$ \\
\hline
\end{tabular}

Cueing effects for trials with correct and incorrect discrimination responses. Shown are marginal means (percentage correct localization accuracy) for validly and invalidly cued objects, separately for trials with correct and incorrect discrimination responses, and the associated main effect of cue, as well as the interaction between correctness of the discrimination response and cue validity. Different rows show different participant inclusion criteria (note that the same participants were included for the inclusion criteria of at least four and five trials per condition). 